Prepared in cooperation with Natural Resources Conservation Service

\title{
Interactive Tool to Estimate Groundwater Elevations in Central and Eastern North Dakota
}

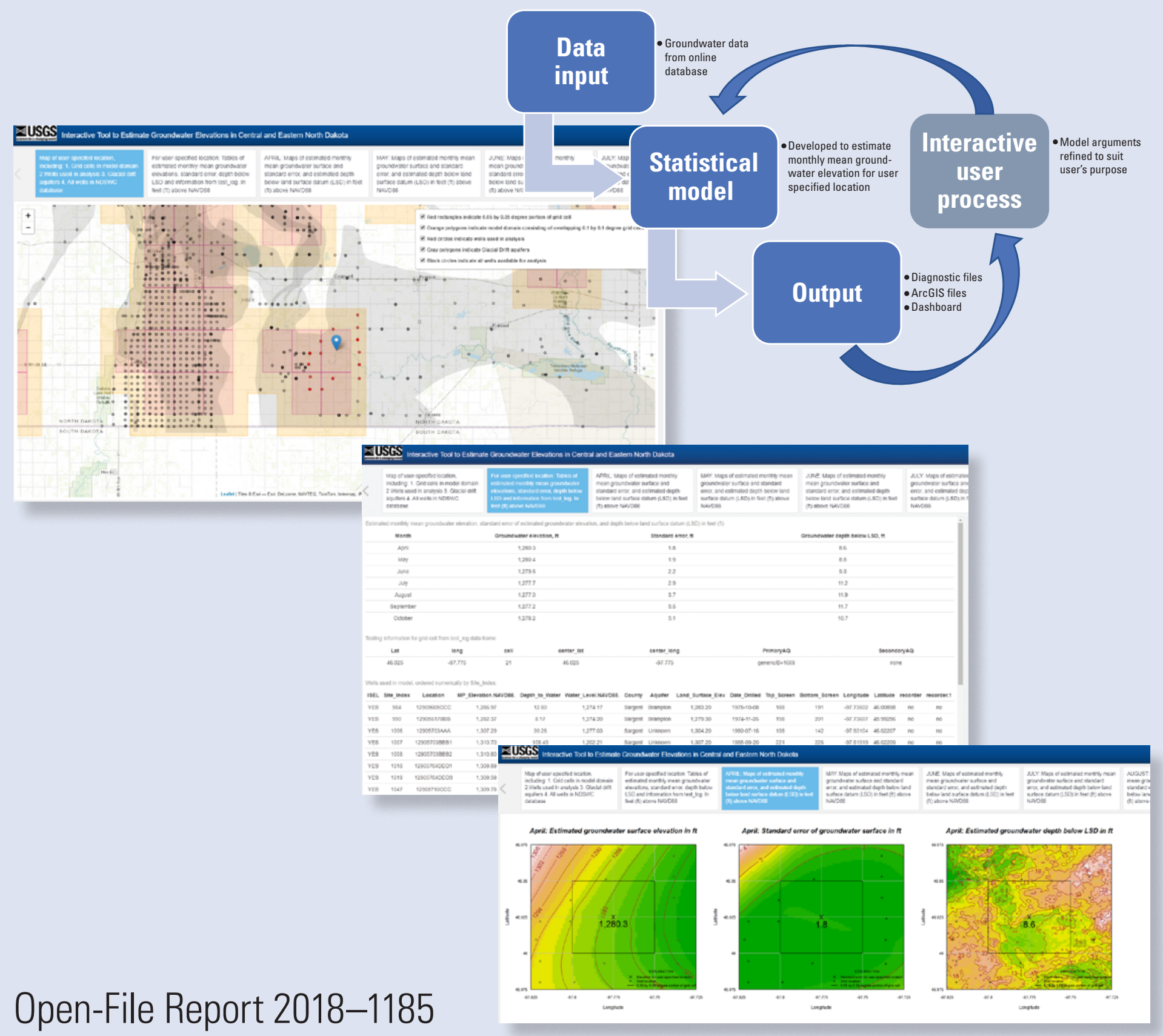

U.S. Department of the Interior

U.S. Geological Survey 
Cover. Screenshots from the NDakGWtool of diagnostic output for a user-specified location with ample data, and diagram showing a general overview of the interactive tool, NDakGWtool. 


\section{Interactive Tool to Estimate Groundwater Elevations in Central and Eastern North Dakota}

By Rochelle A. Nustad, William C. Damschen, and Aldo V. Vecchia

Prepared in cooperation with Natural Resources Conservation Service

Open-File Report 2018-1185 


\title{
U.S. Department of the Interior \\ RYAN K. ZINKE, Secretary
}

\author{
U.S. Geological Survey \\ James F. Reilly II, Director
}

U.S. Geological Survey, Reston, Virginia: 2018

For more information on the USGS - the Federal source for science about the Earth, its natural and living resources, natural hazards, and the environment-visit https://www.usgs.gov or call 1-888-ASK-USGS.

For an overview of USGS information products, including maps, imagery, and publications,

visit https://store.usgs.gov.

Any use of trade, firm, or product names is for descriptive purposes only and does not imply endorsement by the U.S. Government.

Although this information product, for the most part, is in the public domain, it also may contain copyrighted materials as noted in the text. Permission to reproduce copyrighted items must be secured from the copyright owner.

Suggested citation:

Nustad, R.A., Damschen, W.C., and Vecchia, A.V., 2018, Interactive tool to estimate groundwater elevations in central and eastern North Dakota: U.S. Geological Survey Open-File Report 2018-1185, 24 p.,

https://doi.org/10.3133/ofr20181185.

ISSN 2331-1258 (online) 


\section{Acknowledgments}

The feedback provided by Jon Petersen, Natural Resources Conservation Service hydrologist, as the tool was being developed is gratefully acknowledged. Michael Ginsbach, former North Dakota State Water Commission hydrologist, was very helpful in answering questions about data downloaded from the North Dakota State Water Commission's online database.

The authors also acknowledge Kelsey Kolars, former U.S. Geological Survey hydrologist, for her help in compiling the land-surface elevation dataset. 



\section{Contents}

Acknowledgments ……...................................................................................................................

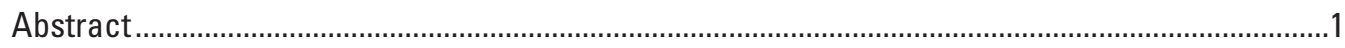

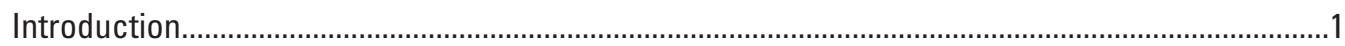

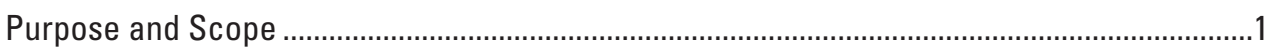

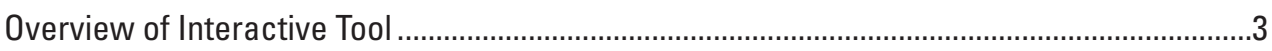

Development of Interactive Tool to Estimate Groundwater Elevations .............................................

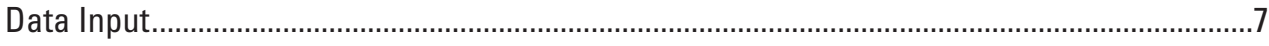

Statistical Model for Estimating Groundwater Elevations .........................................................7

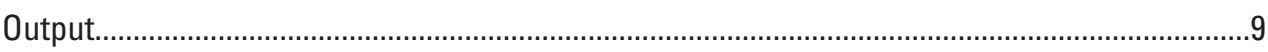

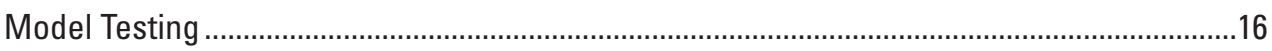

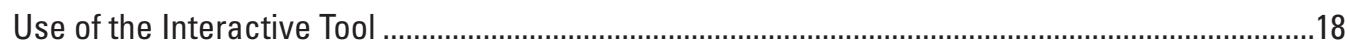

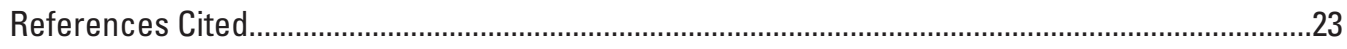

Appendix. R Documentation ..............................................................................................24

\section{Figures}

1. Map showing locations of sites included in the North Dakota State Water Commission database, glacial drift aquifers in North Dakota, and 32 counties in central and eastern North Dakota included in the study area ....................................

2. Diagram showing general overview of the interactive tool, NDakGWtool, to estimate groundwater elevations.

3. Map showing wells in glacial drift aquifers of the study area with at least 1 groundwater elevation measurement from 1960 to 2017 and model domain that consists of 413 overlapping grid cells of 0.1 -degree latitude by 0.1-degree longitude size

4. Screenshots from the NDakGWtool of diagnostic output for a user-specified location with ample data.

5. Example output from the NDakGWtool of ArcGIS files for Cell 21:

Cell 21_specified_location, shape file layer that identifies the user-specified location; Cell 21_Wells, shape file layer that identifies wells used in the analysis, and Cell 21_wl_4.tif is the raster image of estimated groundwater surface for April

6. Screenshots from the NDakGWtool of the static summary file runname_analysis.html, called the dashboard, for Cell 21.

6. Screenshots from the NDakGWtool of the static summary file runname_analysis.html, called the dashboard, for Cell 21

7. Screenshot from the NDakGWtool of diagnostic output for a user-specified location with sparse data.

8. Screenshots from NDakGWtool to illustrate the modification of optional argument spaqnameO..

9. Example of output from the interactive groundwater tool to illustrate modification of an optional argument. 


\section{Tables}

1. R packages, $R$ scripts, $R$ objects, files, and folders required to execute the interactive groundwater tool (NDakGWtool)

2. Arguments used in the wlmodelCell function, which is used for developing a locally weighted regression model for estimating monthly mean groundwater elevations

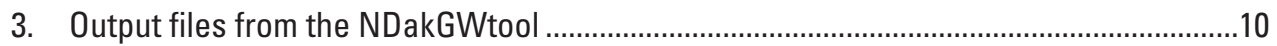

4. Information provided in diagnostic file, runname_wells.csv ...........................................15

\section{Conversion Factors}

U.S. customary units to International System of Units

\begin{tabular}{llll}
\hline & Multiply & By & \multicolumn{1}{c}{ To obtain } \\
\hline & Length & \\
\hline foot (ft) & 0.3048 & meter $(\mathrm{m})$ \\
mile (mi) & 1.609 & kilometer $(\mathrm{km})$ \\
\hline
\end{tabular}

\section{Datum}

Vertical coordinate information is referenced to the North American Vertical Datum of 1988 (NAVD 88).

Horizontal coordinate information is referenced to the North American Datum of 1983 (NAD 83).

\section{Abbreviations}
LWR locally weighted regression
NDSWC North Dakota State Water Commission
NRCS Natural Resources Conservation Service
USGS U.S. Geological Survey 


\title{
Interactive Tool to Estimate Groundwater Elevations in Central and Eastern North Dakota
}

\author{
By Rochelle A. Nustad, William C. Damschen, and Aldo V. Vecchia
}

\section{Abstract}

This report describes an interactive tool (NDakGWtool) in which a statistical model is developed using locally weighted regression to estimate monthly mean groundwater elevations for a specified latitude and longitude, referred to as the "user-specified location." For each user-specified location, seven models are developed for each month from April through October. Localized, high spatial-resolution maps of estimated monthly mean groundwater surface elevations are produced from the models. The tool was evaluated for glacial drift aquifers of the 32-county study area in central and eastern North Dakota. Although groundwater elevations from 1960 to 2017 were available to develop the tool, groundwater elevations from 1995 to 2015 were used for model testing and development of the model domain. There are 413 grid cells of 0.1-degree latitude by 0.1-degree longitude size in the model domain, and the tool produces maps of estimated monthly mean groundwater surface elevations for the cell containing the user-specified location. Additionally, the NDakGWtool produces maps of estimated groundwater depth below land surface and ArcGIS files of estimated groundwater surface elevations and groundwater depth below land surface. The tool is composed of four main components: data input, statistical model, output, and user-interactive process.

\section{Introduction}

The Natural Resources Conservation Service (NRCS) commonly completes hydrologic analyses to determine the effects of proposed agricultural projects, for example, drain tile installation or irrigation withdrawals on shallow groundwater and surface-water resources. A criterion commonly used for their analysis is the 50-percent chance annual probability of inundation for 15 consecutive days during the wetland growing season (U.S. Department of Agriculture, 2015). As a result, in the hydrologic analysis, the NRCS commonly uses groundwater elevations in nearby observation wells to interpolate a seasonal minimum groundwater elevation for the specific location of the proposed project. In North Dakota, an online database hosted by the North Dakota State Water
Commission (NDSWC) provides extensive spatial coverage of groundwater and surface-water data from various sources with about 8.5 million measurements for about 37,000 sites (fig. 1; North Dakota State Water Commission, 2017). Groundwater elevations are available from many types of wells, such as domestic, industrial, irrigation, municipal, observation, and production, but only about 10,000 groundwater sites have at least 1 groundwater measurement. Elevation is defined herein as the altitude of groundwater in feet above the North American Vertical Datum of 1988 (NAVD 88). For sites containing groundwater elevations, there is large variation in measuring periods, many wells have been discontinued or added, and measuring frequencies and seasonal coverage vary extensively. Furthermore, geologic and hydrologic conditions represented by a well network can vary substantially from well to well, and groundwater elevations are affected by spatially and seasonally variable recharge and discharge conditions and pumping. Culling pertinent groundwater elevations from millions of wells in the existing NDSWC database can be an onerous task, and, depending on the project location, availability of nearby wells, and complexity of the groundwater hydrology, it may be difficult to interpolate groundwater elevations in the location of a proposed project. To address this need, the U.S. Geological Survey (USGS), in cooperation with the NRCS, developed an interactive tool (NDakGWtool) to produce estimates of monthly mean groundwater surface elevations for a userspecified location within glacial drift aquifers for a 32-county study area in central and eastern North Dakota (fig. 1). The NDakGWtool will be useful to the NRCS and for future groundwater investigations by the USGS, NDSWC, and other agencies.

\section{Purpose and Scope}

The purpose of this report is to describe the development of an interactive tool (NDakGWtool) used to estimate monthly mean groundwater elevations in central and eastern North Dakota and to illustrate use of the tool. The tool was evaluated for glacial drift aquifers of a 32-county study area in central and eastern North Dakota and the 4 main components of the tool are data input, statistical model, output, and userinteractive process (fig. 2). 


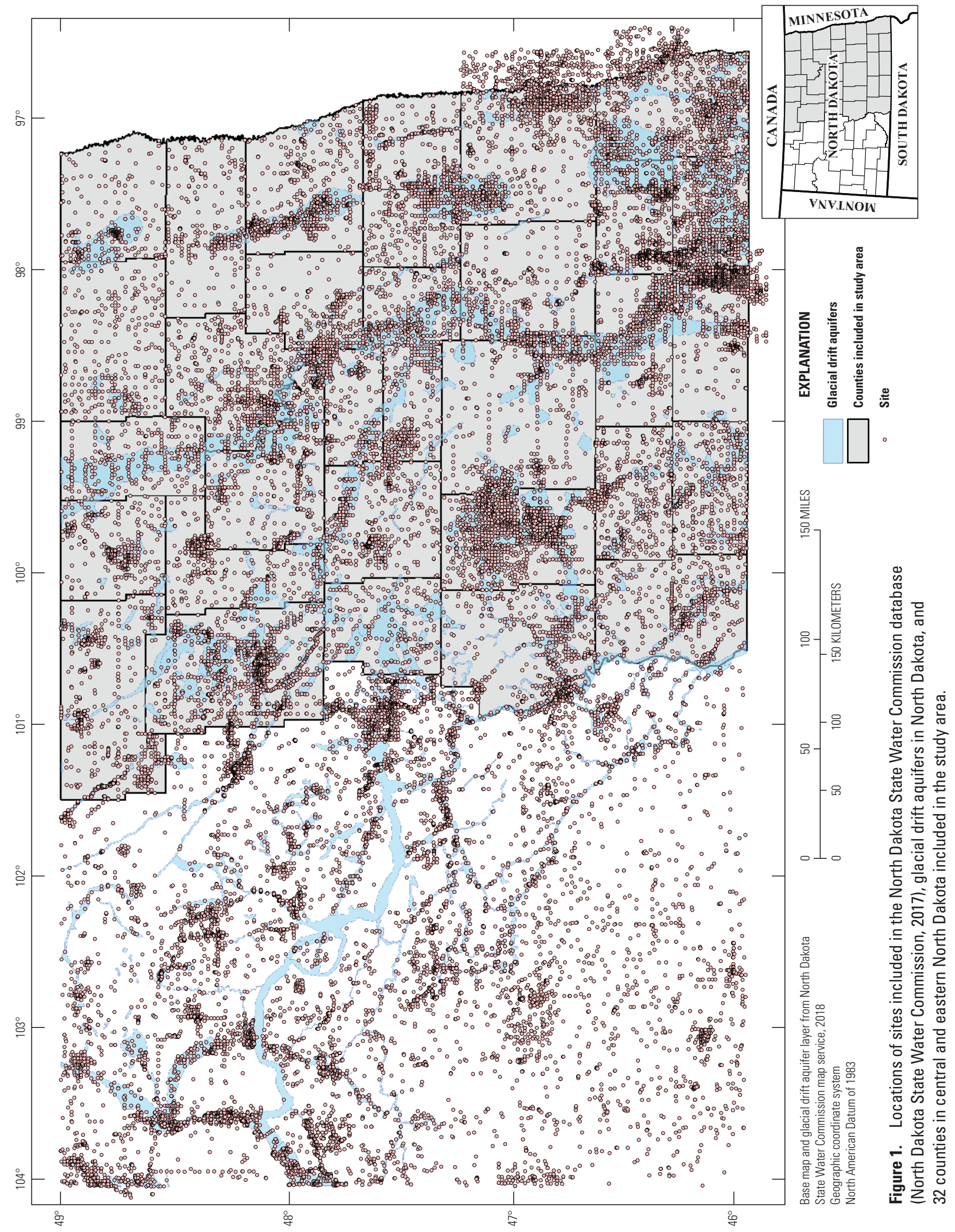




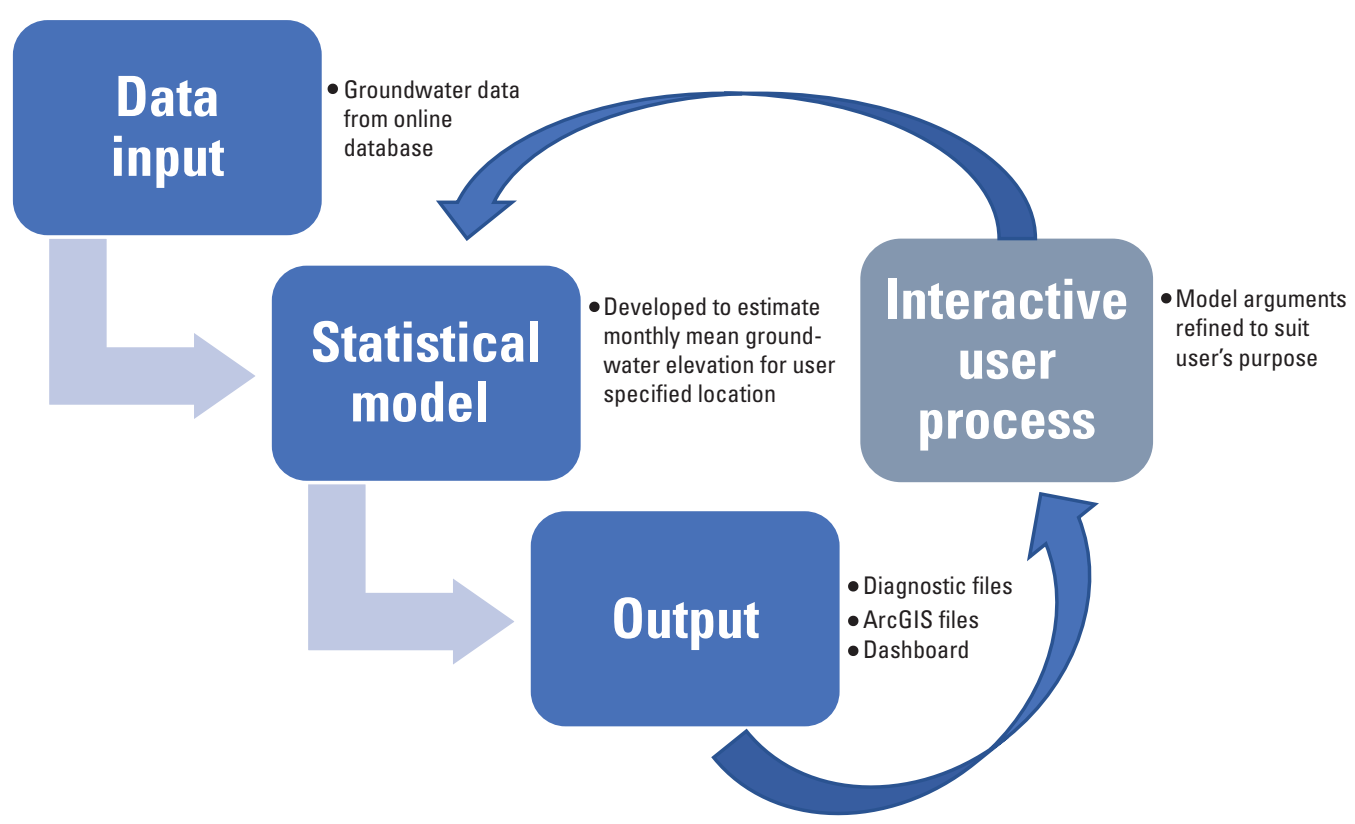

Figure 2. General overview of the interactive tool, NDakGWtool, to estimate groundwater elevations.

\section{Overview of Interactive Tool}

A detailed description of the four main components is provided in subsequent sections, but a general overview of the NDakGWtool is briefly provided here. The NDakGWtool is a statistical model that is developed using locally weighted regression (LWR) to estimate monthly mean groundwater elevations for a specified latitude and longitude, referred to as the "user-specified location."

Temporal and spatial coverages were defined during tool development. Temporal coverage of the tool consists of seven models that are developed for each month from April through October for the user-specified location. For each of these months, localized, high spatial-resolution maps of estimated monthly mean groundwater surface elevations are produced from the models. These maps can represent the water table surface of an unconfined aquifer or the potentiometric surface of a semiconfined aquifer. Consequently, maps of estimated monthly mean elevations will be referred to as "groundwater surface maps." Spatial coverage for the tool was evaluated for glacial drift aquifers of the 32-county study area. The 32-county study area was divided into 5,340 grid cells and, using the center point of each grid cell as the user-specified location, models for each grid cell were evaluated (herein referred to as "model testing"). Although groundwater elevations from 1960 to 2017 were available to develop the tool, groundwater elevations from 1995 to 2015 were used for model testing, and during model testing, the coverage of the tool was narrowed down to grid cells with sufficient data to develop a reliable model. Spatial areas of coverage, meaning output is available from the tool, will be referred to as the "model domain" (fig. 3). There are 413 grid cells in the model domain, and the tool produces maps of estimated monthly mean groundwater surface elevations for the cell containing the user-specified location. Additionally, the NDakGWtool produces maps of groundwater depth below land surface and ArcGIS (Esri, 2018) files of estimated groundwater surface elevations and groundwater depth below land surface.

\section{Development of Interactive Tool to Estimate Groundwater Elevations}

This section provides details on the components of NDakGWtool, development of those components, and use of the tool. The tool was developed in R (R Development Core Team, 2018), a programming and software environment for statistical computing. The tool contains various $\mathrm{R}$ packages, $\mathrm{R}$ scripts, $\mathrm{R}$ objects, files, and folders (table 1 and appendix), but to execute the tool, the primary R script model_run.R is used. Within this script, functions are called to create the groundwater elevation dataset, develop the model, and produce output (table 1). Within the model_run.R script, the code is broken into five sections, referred to as "BLOCKs" and each BLOCK corresponds to the different steps executed in the script. In this report, R functions are given in bold italics and arguments to functions or other $\mathrm{R}$ objects such as data frames are given in italics. An $\mathrm{R}$ data frame is an $\mathrm{R}$ object that holds tabular data, similar to a spreadsheet. A file with an extension ".Rdata" is an $\mathrm{R}$ data format that stores a collection of R objects. 


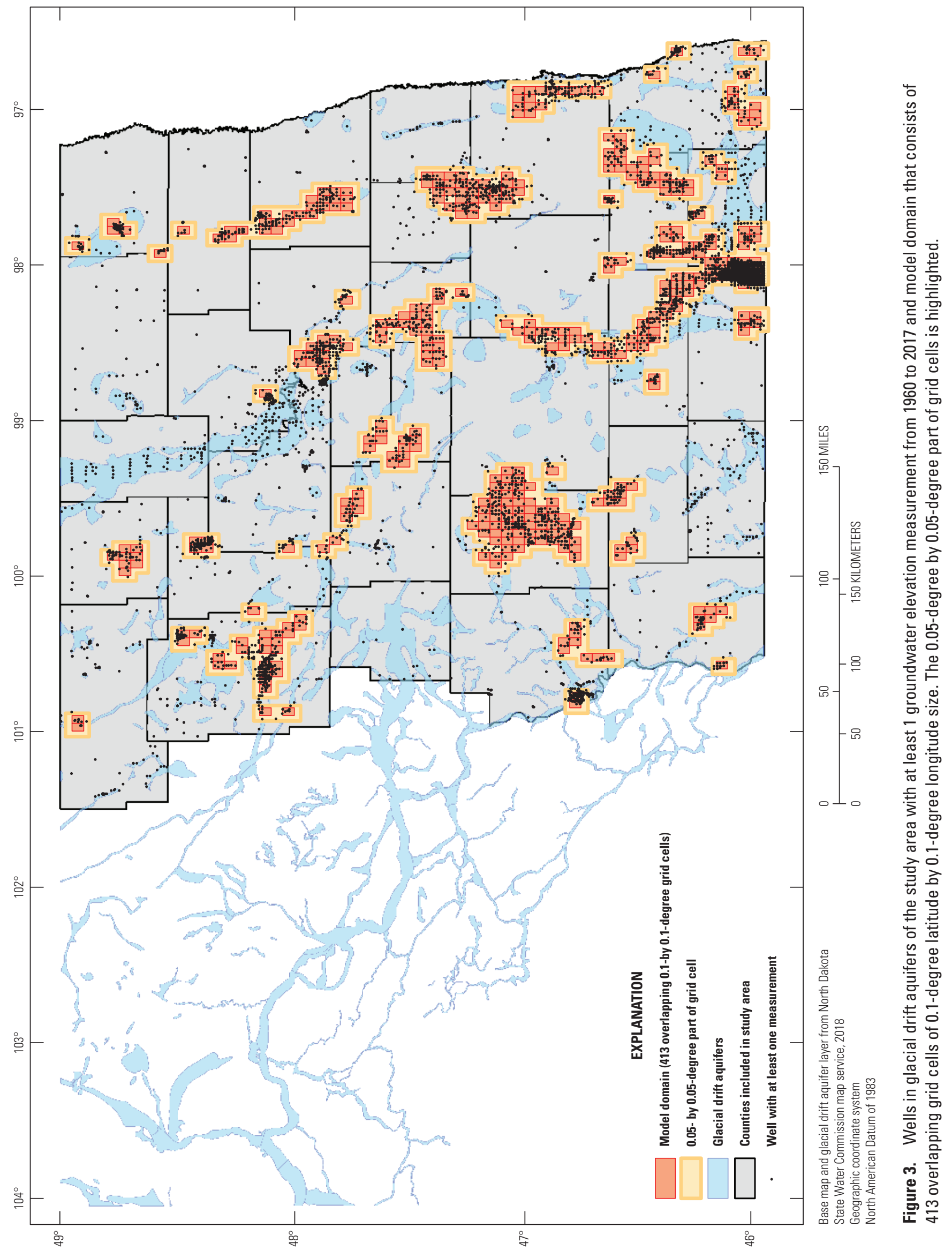




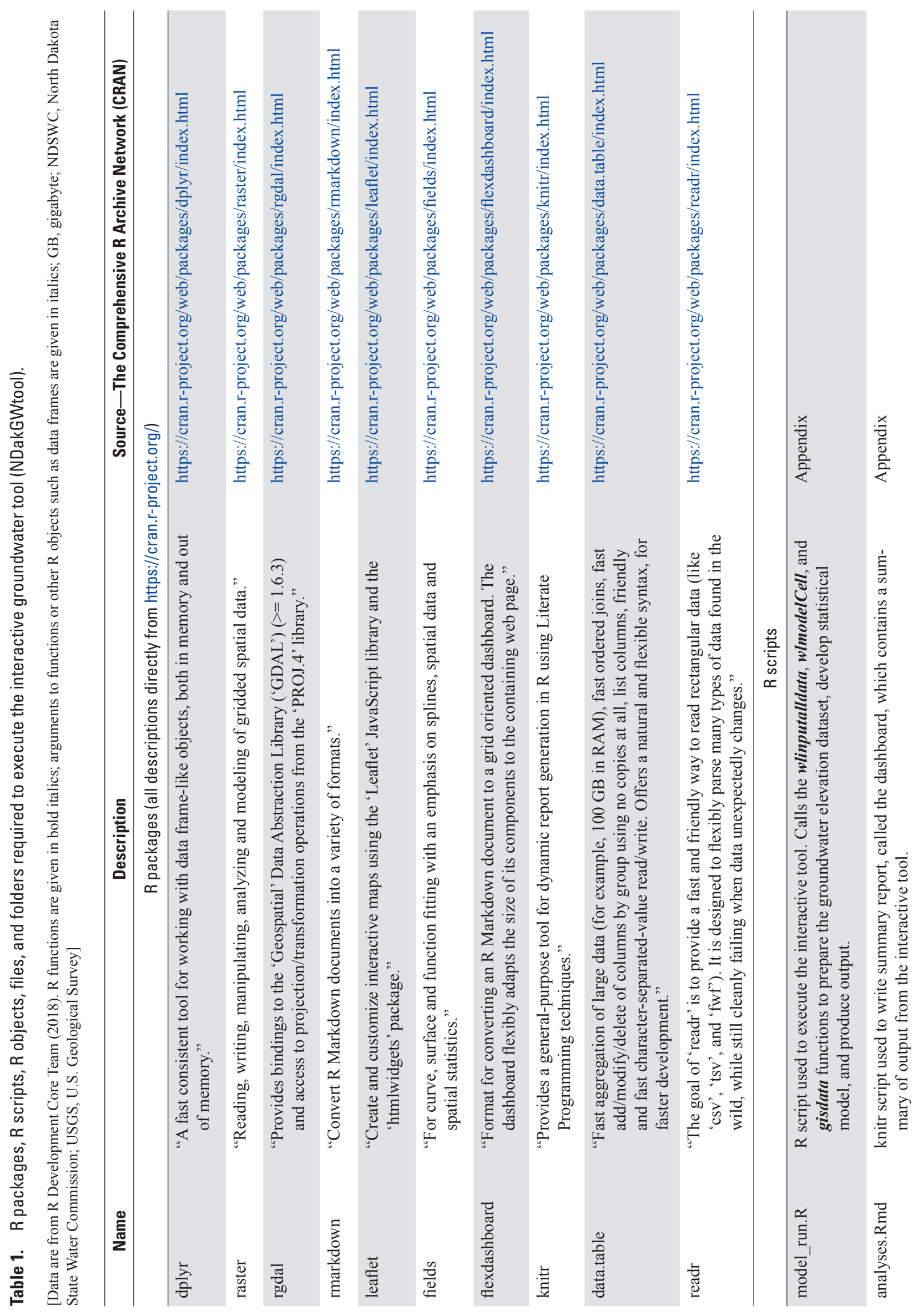




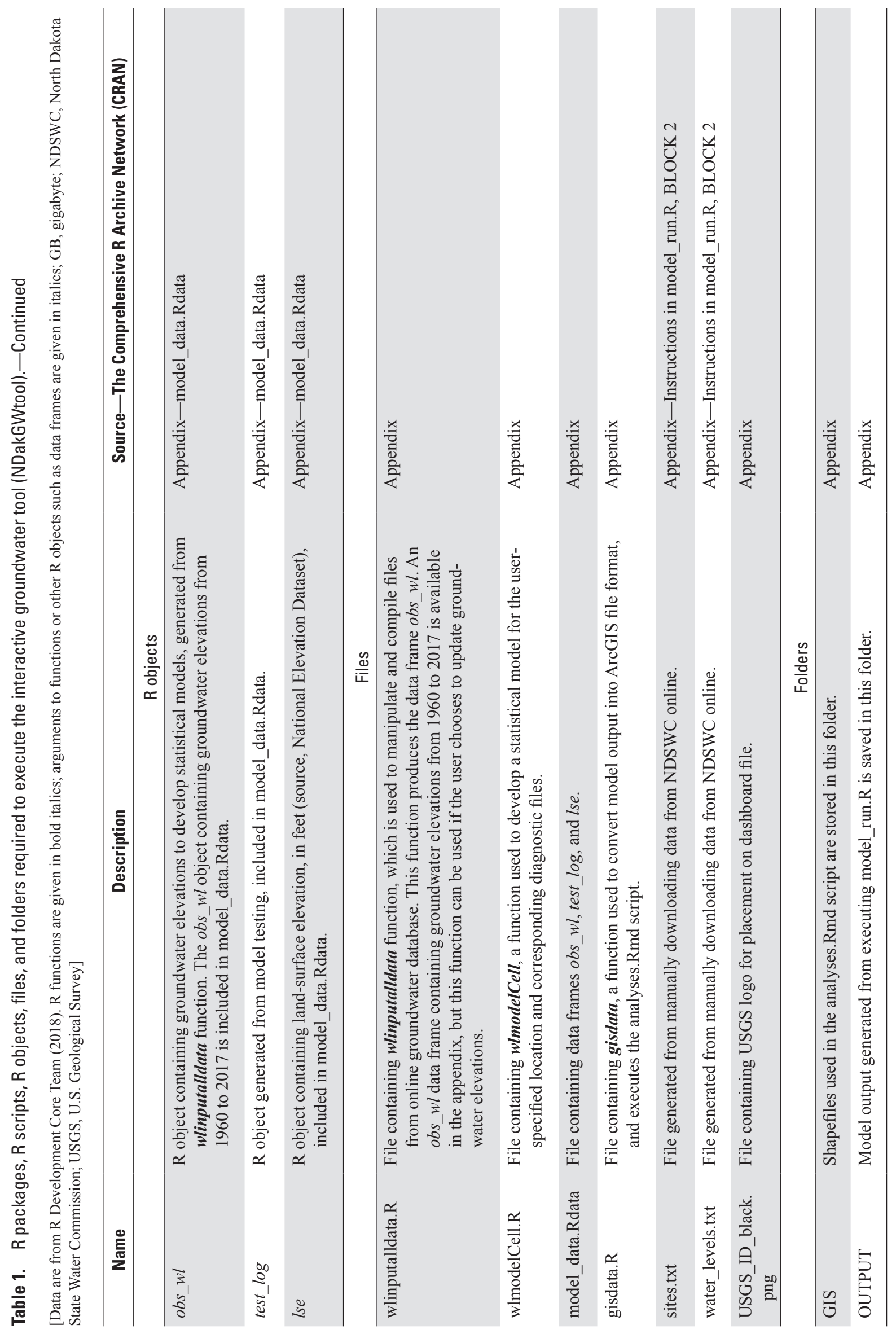




\section{Data Input}

Groundwater elevations and land-surface elevations are required to produce output from the NDakGWtool. Groundwater elevations from 1960 to 2017 are contained in the $o b s \_w l$ data frame, and land-surface elevations for the model domain are contained in the lse data frame (table 1). These two data frames are stored in the model data.Rdata file and are available in the appendix. Instructions to load the model_data.Rdata file are in BLOCK 3 of the model_run.R script (table 1, appendix). Groundwater elevations can be updated by manually downloading the raw dataset and executing the wlinputalldata function to write a new obs $w l$ data frame. Instructions to update groundwater elevations are provided in BLOCK 2 of the model_run.R script (appendix).

The groundwater elevation dataset for this report was downloaded in June 2017 from the NDSWC online database (North Dakota State Water Commission, 2017). All available data were downloaded within the 32-county study area. From these data, two text files were generated, sites.txt and water_levels.txt (table 1). These two files comprise the raw dataset and contain data for groundwater and surface-water sites. The wlinputalldata function combines these two files into one dataset, removes surface-water monitoring sites, selects observation wells in the study area with at least one groundwater measurement since 1960, and eliminates wells missing a measuring point elevation. Groundwater measurements from wells in the raw dataset have measuring frequencies that range from about monthly to continuous, although most wells have a monthly measuring frequency. The measuring frequency generally extends from March or April, depending on the timing of the spring thaw, through October or November. For wells with continuous recorders, the wlinputalldata function selects the last measurement in the month to make them comparable to the wells that have about monthly measurements. Finally, the wlinputalldata function writes selected measurements to the $o b s \_w l$ data frame. The $o b s \_w l$ data frame is the dataset used in developing models for the user-specified location.

Land-surface elevation was acquired for grid cell points in the model domain and used to produce maps of estimated depth of groundwater below land surface. These maps are produced from the gisdata function after models have been developed. Land-surface elevations (1-arc-second resolution) within the model domain were acquired from the USGS National Elevation Dataset and selected for model grid points that correspond to the maps of estimated groundwater elevation (U.S. Geological Survey, 2017). The gisdata function subtracts the estimated groundwater elevation from the landsurface elevation for each grid cell point to compute depth of groundwater below land surface.

\section{Statistical Model for Estimating Groundwater Elevations}

A statistical model is developed for each month from April through October using LWR. In the LWR, groundwater elevations in the $o b s_{-} w l$ data frame are the response variable, and the latitude and longitude of the well locations are the explanatory variable. For the grid cell of the user-specified location, the wlmodelCell function (table 1) selects groundwater elevations, fits an LWR model to those elevations, screens data for outliers, and ultimately produces a localized, high spatial-resolution map of estimated monthly mean groundwater surface elevations. The wlmodelCell function is executed from BLOCK 4 of the model_run.R script (table 1, appendix). In the following paragraphs, the internal computations of wlmodelCell and external requirements, or arguments, for running the function are described.

Internal computations of the wlmodelCell function are completed on available groundwater elevations by grid cell. Grid cells are overlapping blocks of 0.1 by 0.1 degree, about 4.5 by 7 miles (mi), centered on 0.05 - by 0.05 -degree nodes (fig. 3). The grid cell size was chosen to balance the need for spatial detail using available elevations while considering the highly complex and spatially variable nature of groundwater flow. Each grid cell contains 6,400 grid points that are 0.00125 by 0.00125 degree or about 131 by 295 feet (ft). Based on the user-specified location, groundwater elevations for the grid cell in the model domain closest to that location are selected. If the user-specified location lies within the model domain, subsequent steps in the wlmodelCell function are completed. If the user-specified location is outside the model domain the user gets the following error message, "Specified location not in model domain." If a cell does not contain 3 or more observation wells and has at least 30 groundwater elevations for all wells, output is not produced. These are minimum requirements, but most cells had considerably more data.

Once the wlmodelCell function has determined that sufficient data are available for the user-specified location, the next steps involve using the base R function, loess (R Development Core Team, 2018), to fit an LWR model. In the loess function, measured groundwater elevations are defined as the response variable, and the latitude and longitude of well locations are defined as locally fitted explanatory variables. A separate model is created for each month. For estimating monthly mean groundwater elevations for the end of a specified month, measured groundwater elevations for the 12-week (about 3-month) period centered on the last day of the month are used. A third explanatory variable, measurement interval, is defined in the loess function and is the difference between the measurement date and the last day of the specified month. This third explanatory variable was fitted globally using the parametric argument in the loess function (R Development Core Team, 2018) and was used to adjust the measurement dates to represent estimated groundwater elevations for the end of the month. For example, for estimating the monthly mean elevation for April 30, measured groundwater elevations from 
mid-March through mid-June are used. By globally fitting the measurement interval, adjustments are made for variable observation times and short-term trends within the 3-month window. Additional arguments, degree and span, were specified for the loess function (R Development Core Team, 2018). The degree argument refers to the degree of polynomials to be used or the type of model, where a degree $=1$ indicates linear and a degree $=2$ indicates quadratic. The span argument controls the smoothing of the data or the fraction of the data to use for each window, where a span=1 produces the smoothest estimates and smaller values produce more locally variable estimates. Within the wlmodelCell function, degree and span are automatically determined for the loess function based on criteria of density and distribution of wells in the grid cell. A degree $=2$ and span $=0.75$ are indicative of a model developed for a grid cell with high density and even distribution of wells, and degree $=1$ and span $=1$ are indicative of a model developed for a grid cell with low density and (or) a highly clustered well location. For grid cells with spotty or variable seasonal sampling frequencies, the degree and span can vary by month, but they will usually be the same for each month.

Further steps in the wlmodelCell function use the optional loess argument, family, to automatically remove outliers, and once outliers are removed, the base $\mathrm{R}$ function predict.loess is used to estimate groundwater elevations for each point in the model grid cell (R Development Core Team, 2018). Outliers are common in groundwater elevations and are caused by several factors: if a topographic map is used to determine well location and measuring point, accuracy of the groundwater measurement may be poor; some wells demonstrate highly localized behavior and, as such, the groundwater measurement may not be representative of a spatially coherent and relatively smooth water surface; data transcription errors for some groundwater measurements may result in incorrect values; or extreme events such as frozen or partially frozen soils, local snowmelt, recharge, or precipitation may result in measurements that are not representative of typical conditions. These types of outliers were removed to suit the needs of the NRCS for their hydrologic analysis and to improve model stability. An automated screening procedure was used to detect and remove as many outliers as possible by fitting the initial model using the family = "symmetric" argument in the loess function. The family = "symmetric" argument uses the robust Mestimation method, which is a common and effective method for removing outliers (Cleveland and others, 1992). Outliers, specifically defined as elevations with an absolute value of the residual greater than 2.5 standard errors, are removed and the model is fitted a second time with the family = "symmetric" option. Additional outliers, if there are any, are once again removed. Finally, groundwater elevations remaining after the two outlier screenings are used to fit a final model using the family = "gaussian" argument or ordinary least squares. After removing potential outliers and fitting the final model to groundwater elevations for the grid cell, the function predict. loess is used to estimate monthly mean elevations and the standard error of the estimated elevations for each grid point contained within the grid cell of the user-specified location (table 1, appendix).

As previously mentioned, the user executes the wlmodelCell function in BLOCK 4 of the model_R script. Available arguments to the wlmodelCell function include 3 required arguments and 3 optional arguments. Required arguments include runname, a prefix attached to output files; latsp, the user-specified latitude for the location of interest, in decimal degrees; and lonsp, the user-specified longitude for the location of interest, in decimal degrees (table 2). For user convenience, the required arguments for the wlmodelCell function can be populated in the dummy variables of BLOCK 4 in model_R.script rn for runname (line 89); lat for latsp (line 92); and lon for latsp (line 93).

The first optional argument, spaqname0, is included in the wlmodelCell function to allow the user to interactively modify the set of wells used in the models (table 2). If the user chooses to define the spaqname 0 argument, this also is in BLOCK 4 of the model_R.script, but the user populates this argument directly within wlmodelCell function, on line 100. This optional argument may be helpful to the user because grid cells can contain a mixture of wells with groundwater elevations from different aquifers, and, moreover, wells in the same aquifer can represent a mixture of unconfined or semiconfined layers, even if the wells are near one another. If spaqname 0 is omitted from the argument list or spaqname $0=$ "default" is specified, the default value described later in the "Model Testing" section is used.

The argument spaqname 0 is a character variable with the following format:

$$
\text { spaqname0 = "aqname/spdepth/spbadwells/splevels" }
$$

where

aqname $=$ "generic" indicates that all wells (regardless of aquifer name) should be included;

aqname $=$ "Oakes" (or any other user-specified aquifer name) indicates that only wells in the Oakes aquifer (or any other user-specified aquifer name) should be included;

spdepth $=$ " $D>d$," where $d$ is a user-specified numeric constant, indicates that only wells with screen-interval depths $(D)$ greater than $d \mathrm{ft}$ should be included;

spdepth $=$ " $D<d$," where $d$ is a user-specified numeric constant, indicates that only wells with $D$ less than $d \mathrm{ft}$ should be included;

spbadwells $=$ " $B=i 1, i 2, \ldots, i K$," where $i 1, i 2, \ldots, i K$ are $K$ $(K \geq 1)$ user-specified well numbers, indicates that wells $(B)$ with the userspecified well numbers should be excluded;

splevels $=$ " $L>w$," where $w$ is a user-specified numeric constant, indicates that only groundwater elevations $(L)$ greater than $w \mathrm{ft}$ should be included; and 
splevels $=$ " $L<w$," where $w$ is a user-specified numeric constant, indicates that only $L$ less than $w \mathrm{ft}$ should be included.

The aquifer name (aqname) must be specified, but the modifiers (terms involving $D, B$, or $L$ separated by “/”) are optional. The first two characters of each modifier (" $D>$ " or " $D<$ ", " $B=$ ", " $L>$ " or " $L<$ ") must be exactly as specified, with capital letters and no spaces, and the well indices are separated by commas (no spaces). For example,

spaqname0 = "generic" selects all groundwater elevations regardless of aquifer name;

spaqname $0=$ "generic $/ D>100 "$ selects only wells with screen-interval depths greater than $100 \mathrm{ft}$;

spaqname $0=$ "generic $/ D>100 / B=2417,2425$ " excludes wells with index number 2417 or 2425 (in addition to $D>100$ ); spaqname0 = "Spiritwood" selects only wells in the Spiritwood aquifer; and

spaqname $=$ "Spiritwood $/ L>1300$ " selects only wells in the Spiritwood aquifer and uses only groundwater elevations greater than $1,300 \mathrm{ft}$.
The other two optional arguments, yrbeg and yrend (table 2), can be used to override the default period of 19952015 used to estimate the groundwater elevations; for example, specifying $y$ rbeg $=2005$ and $y$ rend $=2015$ uses groundwater elevations for 2005-15 to fit the model.

\section{Output}

Three types of output are produced from the NDakGWtool and include (1) diagnostic files, in .pdf and .csv file formats; (2) ArcGIS files, in .tif and shp file formats; and (3) a static summary file, in .html file format, called the dashboard (table 3). Diagnostic output is generated by the wlmodelCell function, and files are produced by running BLOCK 4 of the model_run.R script. The other two types of output are generated by the gisdata function (table 1, appendix) and are produced by running BLOCK 5 of the model_ run.R script. The runname argument defined by the user in BLOCK 4 of the model_run.R script is attached as a prefix to all output file names. Output files are stored in a folder labeled

Table 2. Arguments used in the wlmodelCell function, which is used for developing a locally weighted regression model for estimating monthly mean groundwater elevations.

[Arguments to functions or other R objects such as data frames are given in italics]

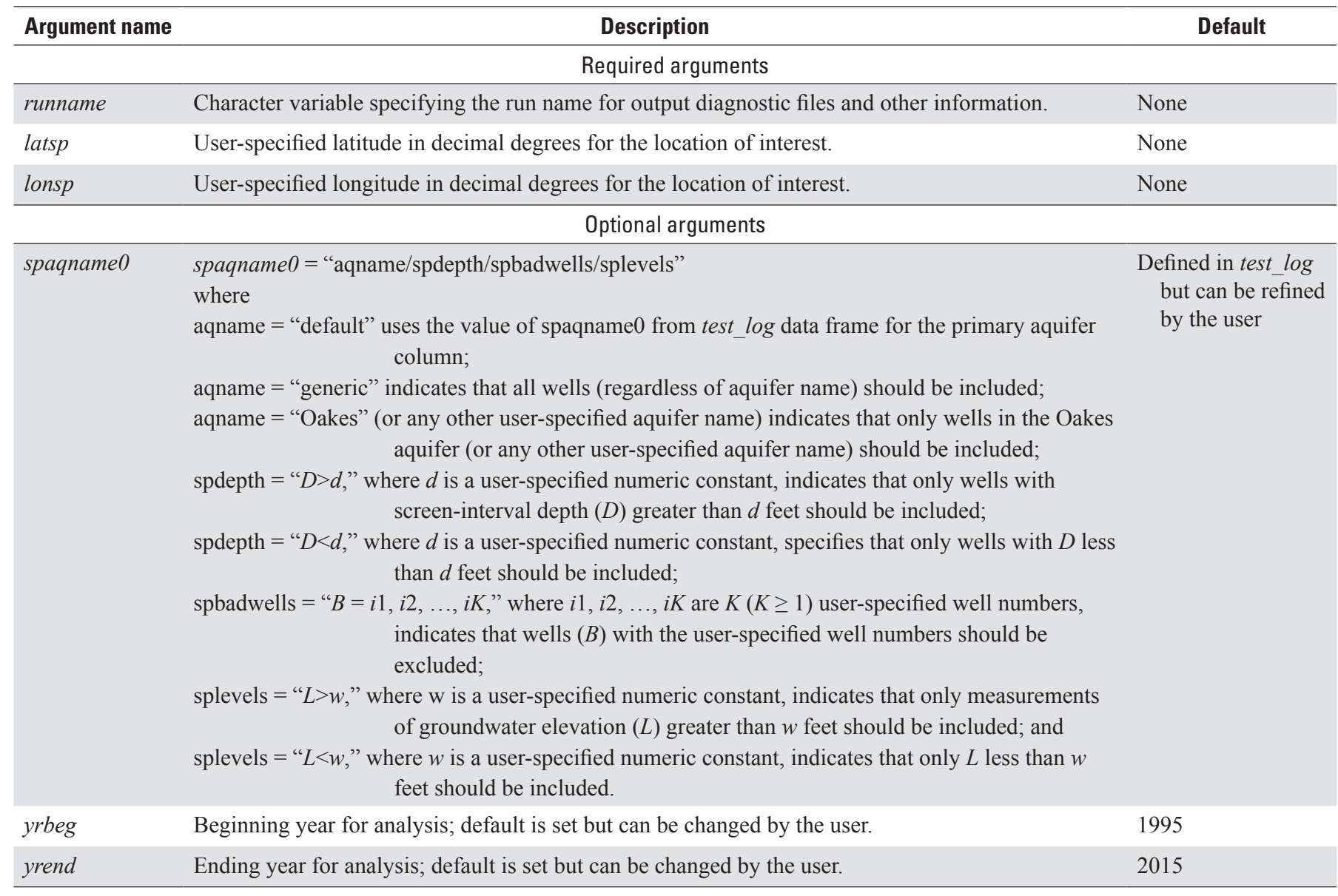




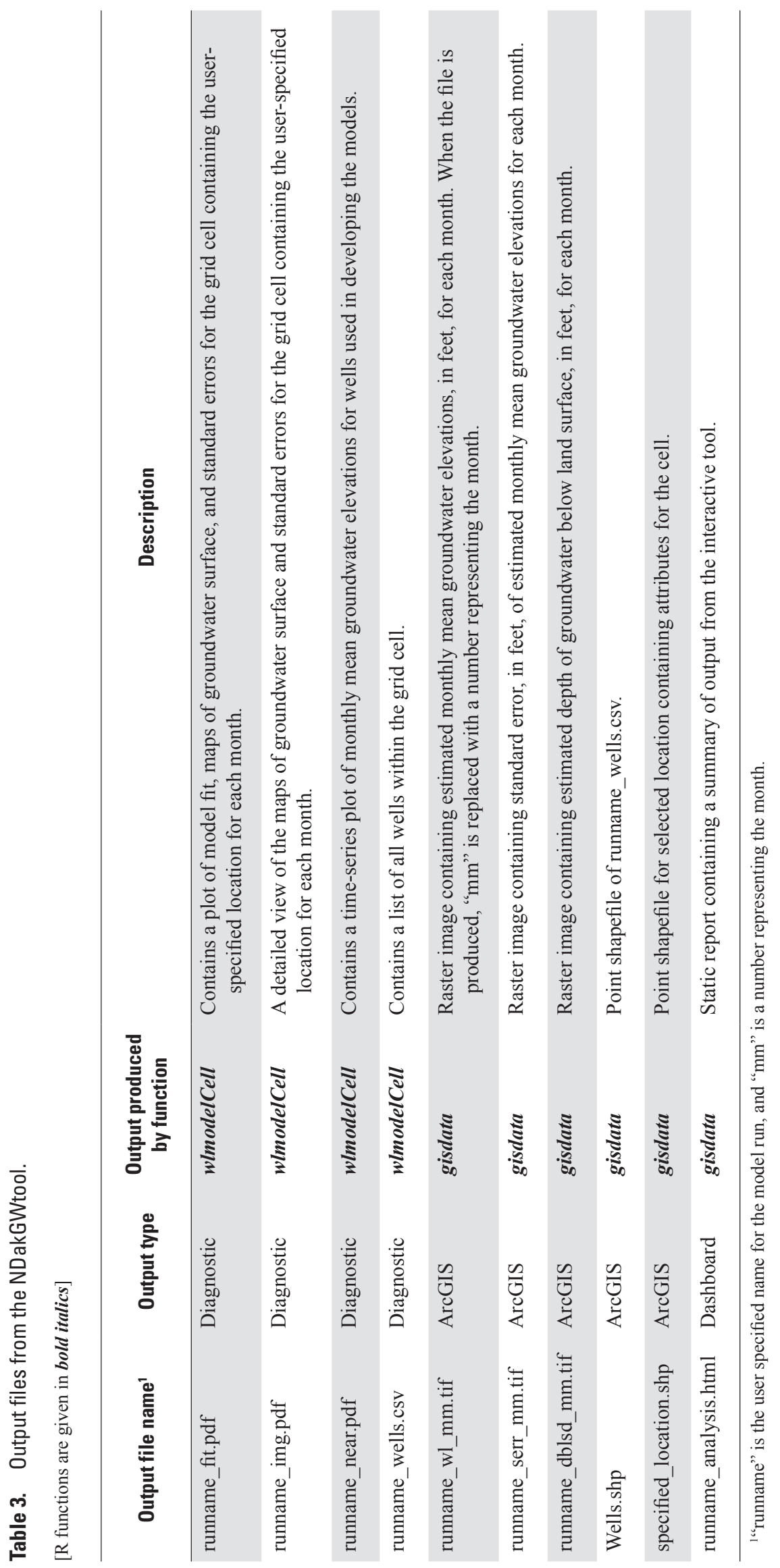


with the runname prefix in a subdirectory of the "OUTPUT" folder.

Output for the center point of Cell 21, the user-specified location of lats $p=46.025$, lons $p=-97.775$, a location with ample data, will be used to illustrate output from the tool (fig. 4). Grid cell numbers were given to the 413 grid cells in the model domain and are included in the test_log data frame (appendix). In this example, the cell number was used for the runname argument, but any name can be specified. Specifically, in BLOCK 4 of the model_run.R script, dummy variables rn, lat, and lon in lines 89, 92, and 93, respectively, were used to populate arguments for the wlmodelCell function of runname, latsp, and lonsp as follows: $\mathrm{rn}=$ "Cell 21"; lat=46.025; lon=-97.775 (appendix). To produce output for this example, BLOCK 4 and BLOCK 5 in the model_run.R script were executed.

Diagnostic files include (1) runname_wells.csv, a file containing a table of the wells in the user-specified grid cell available for developing the model (fig. 4A); (2) runname_near.pdf, a file containing a time-series plot of monthly mean groundwater elevations for wells used in developing the model (fig. 4B); (3) runname_fit.pdf, a file containing a plot of model fit, maps of estimated monthly mean groundwater surface and standard errors for the grid cell containing the user-specified location for each month (fig. 4C); and (4) runname_img.pdf, a detailed view of the map of the estimated monthly mean groundwater surface and standard errors (fig. 4D). These files are described in detail below. In figures $4 C$ and $4 D$, April is used for an example of the plots, but plots for each month are produced in the files.

The runname_wells.csv file contains a list of all wells within the user-specified grid cell. Wells used in the model are listed first and are ordered from closest to farthest from the user-specified location (latsp, lonsp). Wells not used in the model are listed last and are indicated by "NO" in the column heading titled "ISEL" (fig. $4 A$, table 4).

The runname_near.pdf file (fig. $4 B$ ) includes two plots per well for wells used in developing the model (each well for which ISEL = "yes") and a hypothetical "well" at the userspecified location (latsp, lonsp). For figure $4 B$, the first four wells used in the analysis are shown. User-specified arguments runname, latsp, and lonsp are identified in the title of the plots, and the well order is the same as in the runname_wells.csv file. For each well, the first or left-hand plot shows the estimated monthly means for the end of each month. The value of the highest and lowest monthly mean groundwater elevation is explicitly labeled. The 98th and $2 \mathrm{~d}$ percentile of the estimated groundwater elevations also are identified, and most of the measurements should lie within these upper and lower prediction limits. For the hypothetical well, only estimated groundwater elevations are plotted. Note that groundwater elevations removed through the outlier screening are not shown in these plots. The second (right-hand) plot contains the estimated monthly mean groundwater elevations and prediction limits

\section{A}

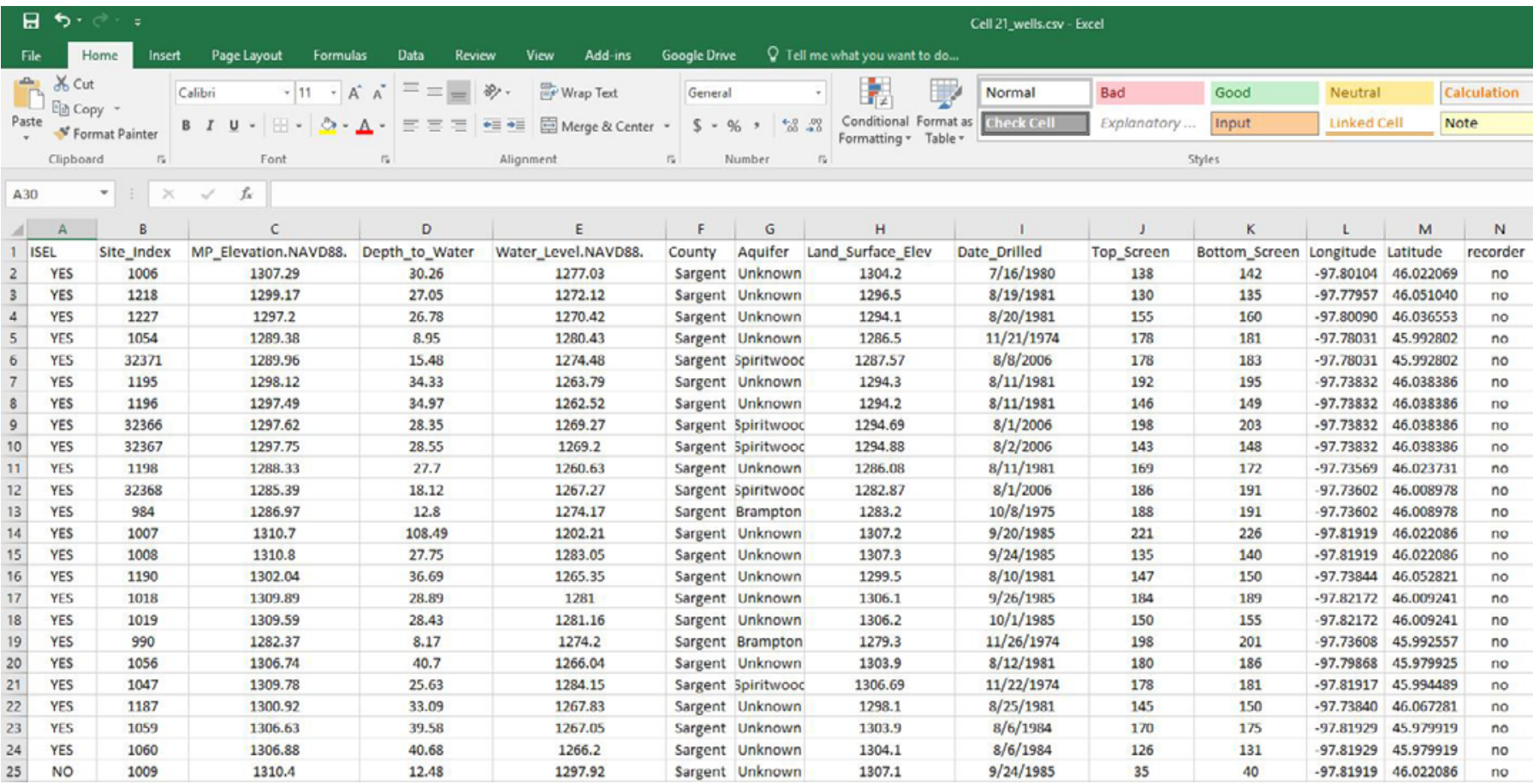

Figure 4. Screenshots from the NDakGWtool of diagnostic output for a user-specified location with ample data. Arguments specified in wlmodelcell function: lats $=46.025$, lons $p=-97.775$, runname = Cell 21, and spaqname 0 = "default." $A$, runname_wells.csv, a file containing a table of the wells in the user-specified grid cell available for developing the model. 

Estimated and measured groundwater elevations for wells used in analysis
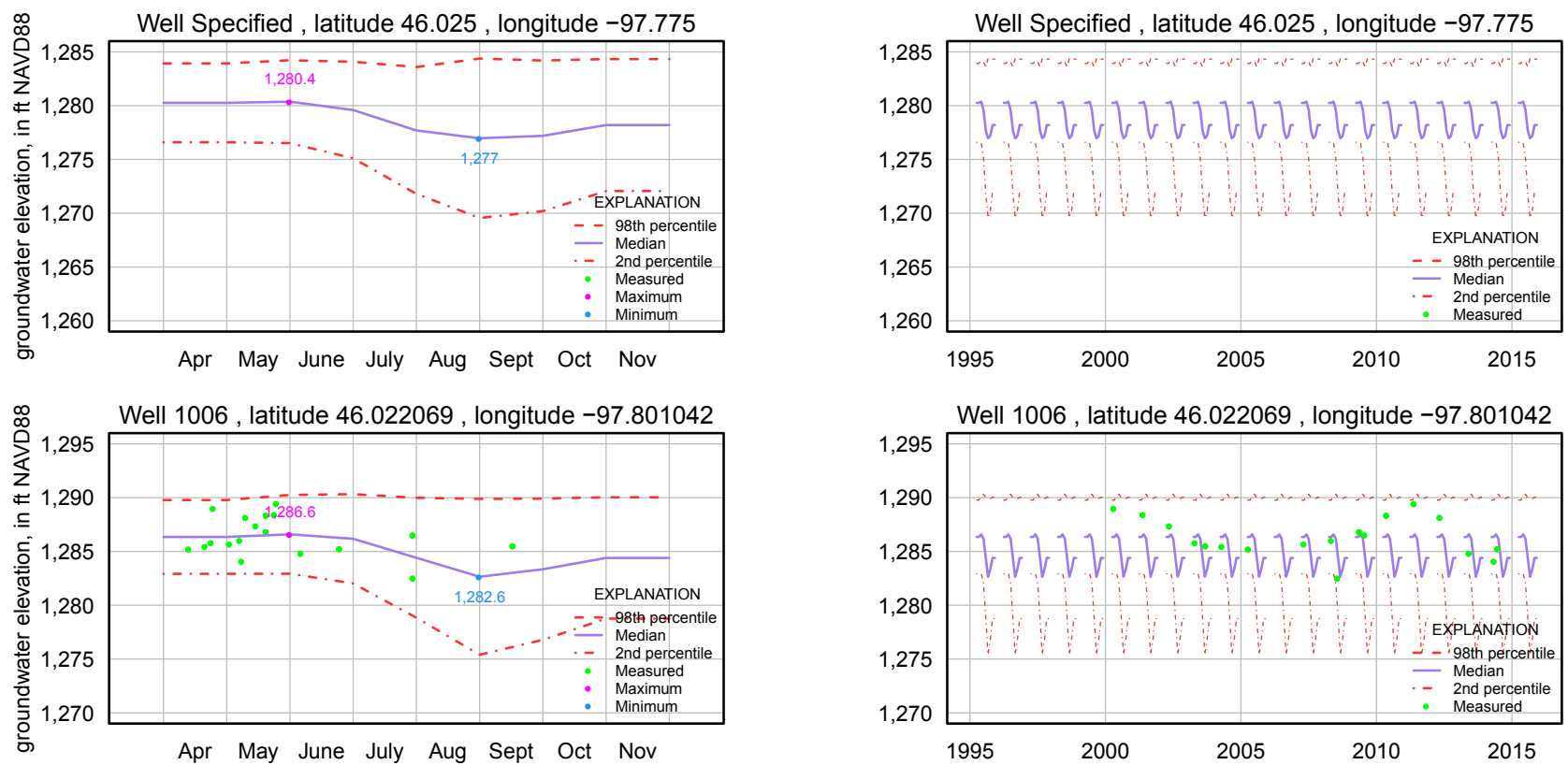

Well 1006 , latitude 46.022069 , longitude -97.801042
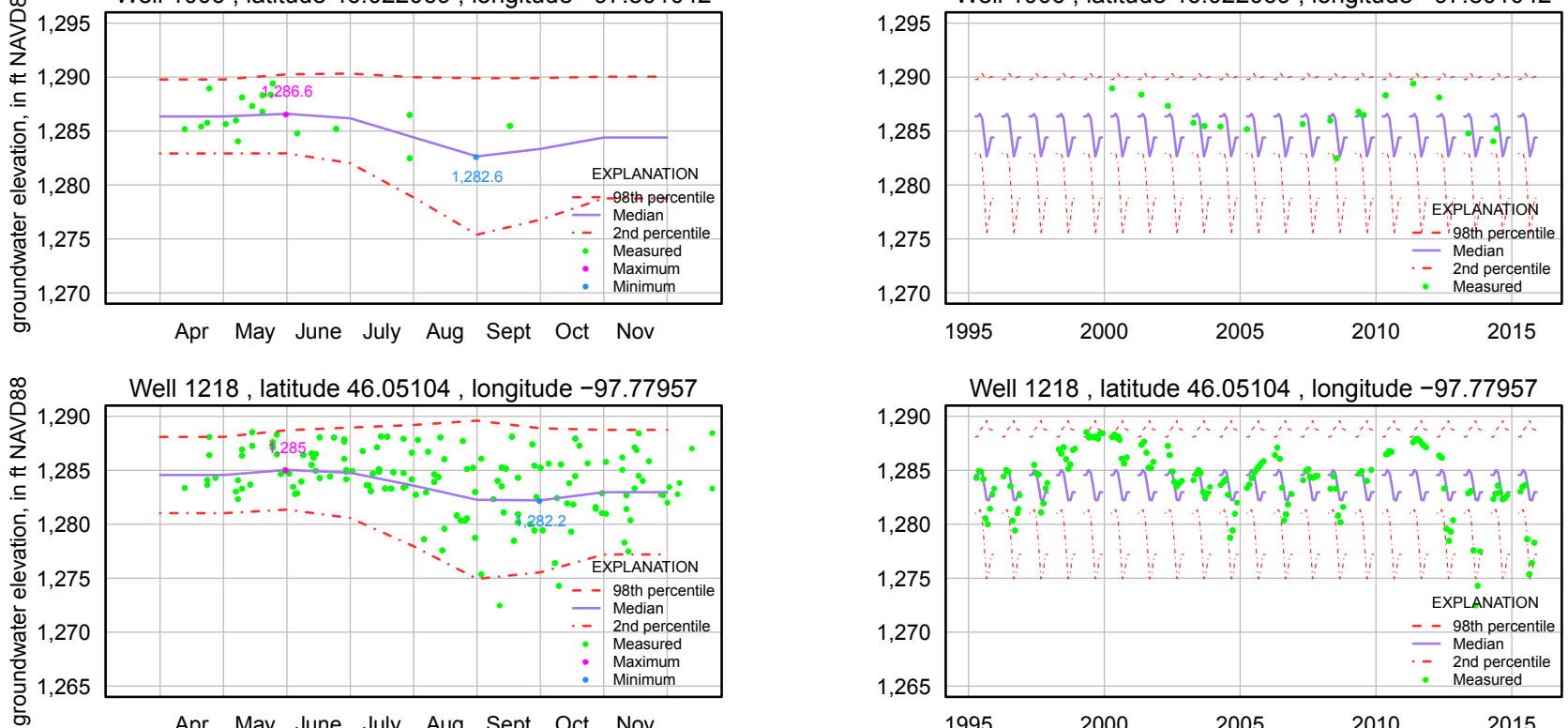

Well 1218 , latitude 46.05104 , longitude -97.77957
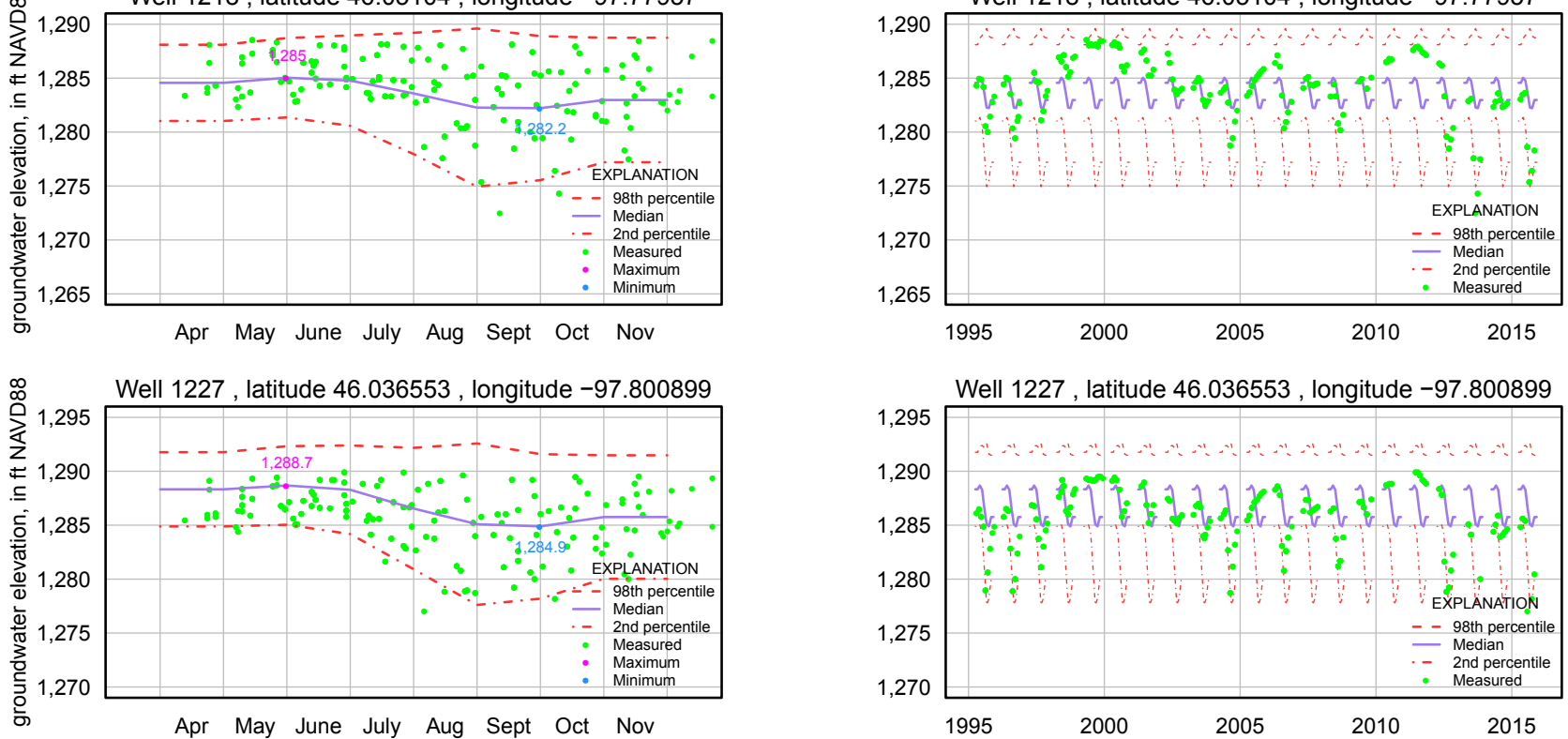

Well 1227 , latitude 46.036553 , longitude -97.800899
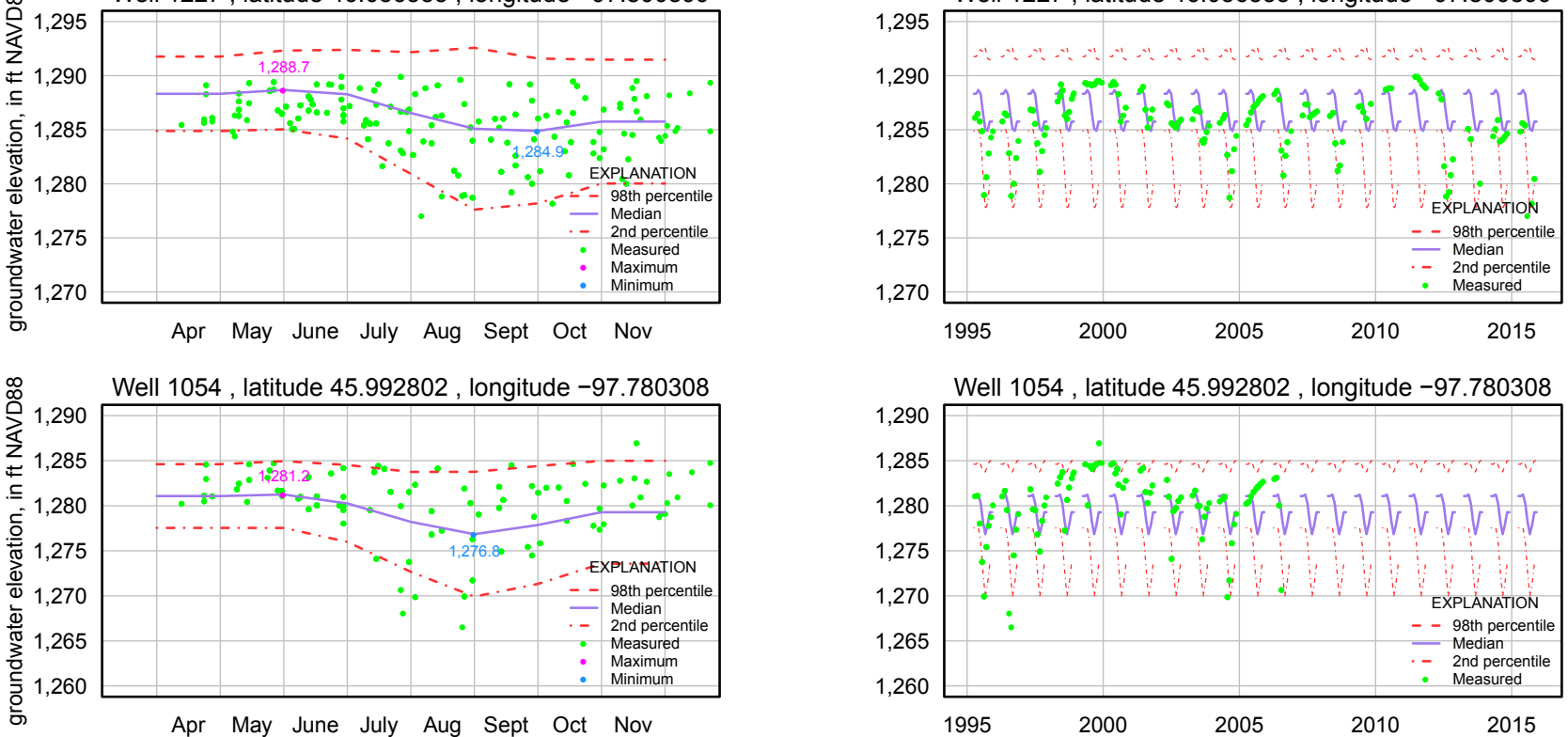

Well 1054 , latitude 45.992802 , longitude -97.780308

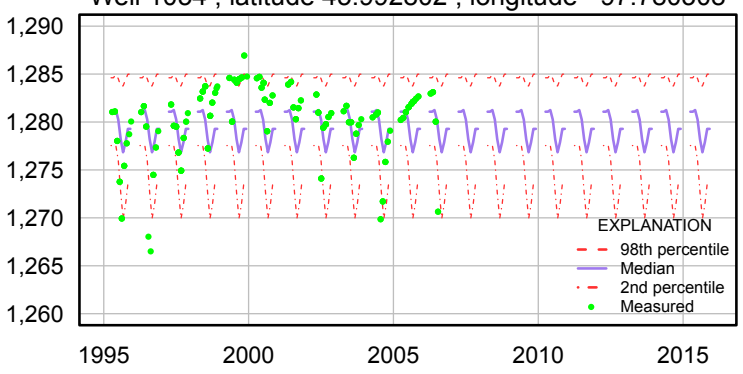

Figure 4. Screenshots from the NDakGWtool of diagnostic output for a user-specified location with ample data. Arguments specified in wlmodelcell function: lats $p=46.025$, lons $p=-97.775$, runname = Cell 21, and spaqname0 = "default.-Continued. $B$, runname_near.pdf, a file containing a time-series plot of monthly mean groundwater elevations for wells used in developing the model (a subset of wells is presented here). 
from the left-hand plot, repeated for each year used for the water-level model, along with the same measured groundwater elevations used to fit the model to produce the monthly averages. These plots can be examined to identify any potential long-term trends or problems from nonoverlapping or missing periods of record for different wells.

The runname_fit.pdf file provides an overview of the model with a set of three plots for each month: a plot of model fit; a map of the estimated monthly mean groundwater surface; and a map of the standard errors of the estimated groundwater surface (fig. 4C). In the left-hand plot, estimated versus measured groundwater elevations, along with the line of equality plus or minus one standard error, are plotted to show model fit. From this plot, irregularities such as clustering of points above or below the line of equality; highly nonsymmetrical distribution of points, for example, many more points below the line than above; or other irregularities can be identified. If the user identifies irregularities, the user can refine the process to correct these irregularities. This is illustrated below in the "Use of the Interactive Tool" section. Additional information about the model is provided in the text above the three plots and includes the number of outliers removed through the outlier screening, the number of measurements left after outlier screening, the number of wells used in the model, and the span and degree of the LWR model. The middle plot is a map of the estimated monthly mean groundwater surface elevation for the 0.1 - by 0.1 -degree cell and a black box highlights the center 0.05 - by 0.05 -degree part of the cell. The $\mathrm{X}$ identifies the userspecified location for the model run (latsp, lonsp), and circles represent the location of the wells included in the analysis.
This map allows a cursory examination of the shape of the groundwater surface and the number and location of wells that had groundwater elevations for a given monthly window. Note that the number and locations of the wells shown in these plots can differ among months, especially if wells have spotty seasonal coverage. Also note that the range in groundwater surface elevation is indicated above each plot and differs from month to month. The right-hand plot is a map of the standard errors for estimated groundwater elevations provided in the groundwater surface map.

A more detailed view of the estimated monthly mean groundwater surface map and standard errors are shown in the runname img.pdf file (fig. $4 D$ ). In this file, all wells used in the model, regardless of month, are shown and the well index numbers are given. Also, groundwater elevation ranges and colors are the same for every month, so groundwater surface elevations in different months can be compared.

ArcGIS files include raster files of estimated monthly mean groundwater surface elevations, standard error (in feet) of estimated groundwater surface, and estimated depth of groundwater below land surface (in feet) for each month for the grid cell of the user-specified location (fig. 5). Note, only output for estimated groundwater surface elevation for the month of April is shown on figure 5. There are 21 .tif files produced for each model run; a tif file is produced for each of the 7 months, April-October, for 3 parameters: (1) estimated groundwater surface elevations, (2) standard error of estimated groundwater surface, and (3) estimated groundwater depth below land surface. In addition, two shapefiles (.shp) that

\section{C}

Cell 21 (latsp=46.025, lonsp=-97.775) - Model fit and estimated monthly mean groundwater surface and standard errors for Apr $1995-2015$
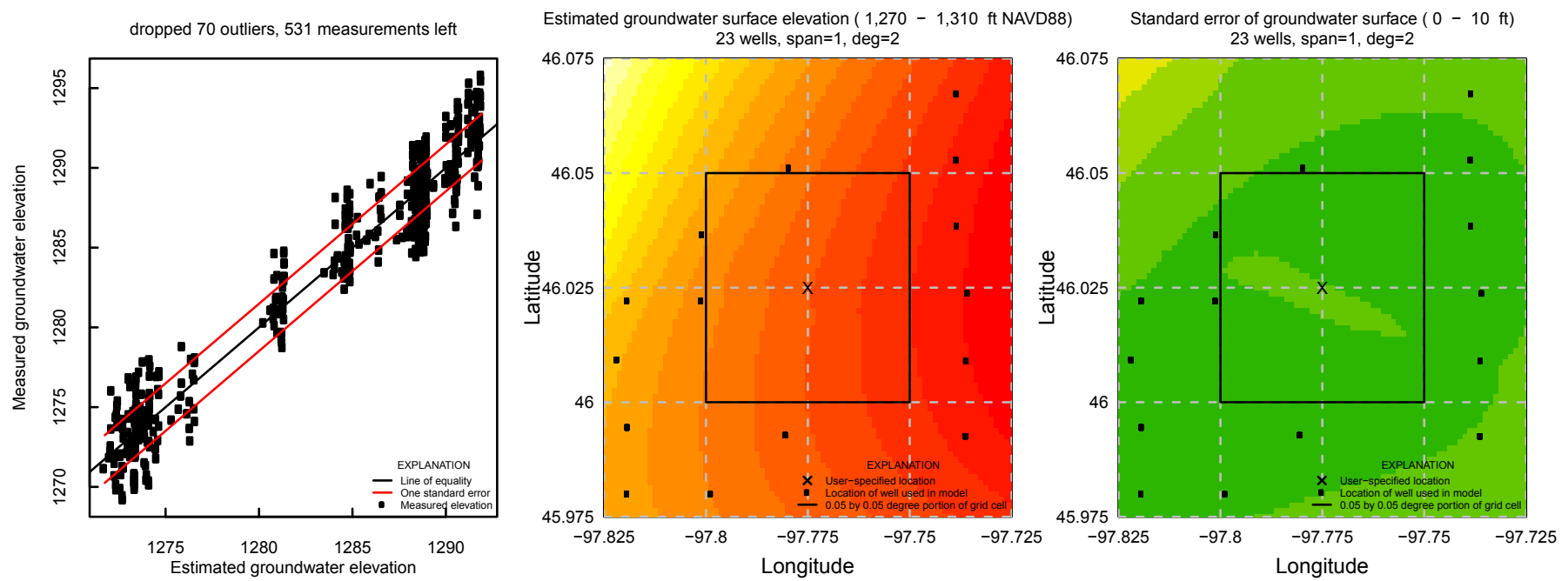

Figure 4. Screenshots from the NDakGWtool of diagnostic output for a user-specified location with ample data.-Continued. Arguments specified in wlmodelcell function: lats $p=46.025$, lonsp=-97.775, runname = Cell 21, and spaqname0 = "default. $C$, runname_fit.pdf, a file containing a plot of model fit, maps of estimated monthly mean groundwater surface and standard errors for the grid cell containing the user-specified location for each month (only April is shown here). 
D

Cell 21 (latsp=46.025, lonsp=-97.775) - Estimated monthly mean groundwater surface and standard errors for Apr $1995-2015$
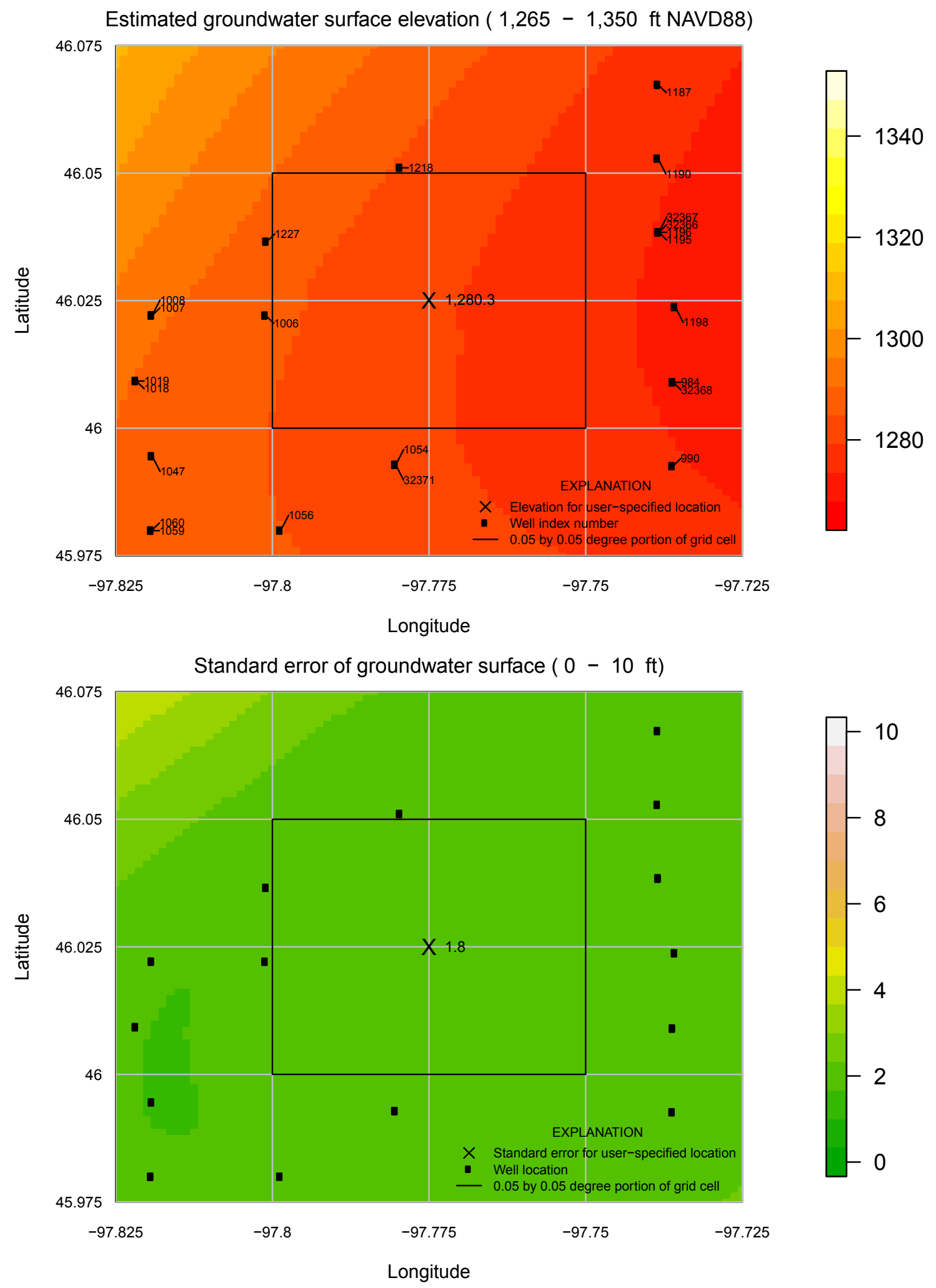

Figure 4. Screenshots from the NDakGWtool of diagnostic output for a user-specified location with ample data.-Continued. Arguments specified in wlmodelcell function: lats $p=46.025$, lons $p=-97.775$, runname $=$ Cell 21 , and spaqname $0=$ "default. $D$, runname_img.pdf, a file containing a more detailed view of the map of estimated monthly mean groundwater surface and standard errors (only April is shown here). 
Table 4. Information provided in diagnostic file, runname_wells.csv.

\begin{tabular}{|c|c|}
\hline Column name & Description \\
\hline ISEL & Identifies if the well is used in the development of the water level model. \\
\hline Site_Index & Well name. \\
\hline MP_Elevation.NAVD88. ${ }^{1}$ & Measuring point elevation for the well. \\
\hline Depth_to_Water & $\begin{array}{l}\text { Depth to water calculated for the first observation for the well by subtracting Water_Level.NAVD88. } \\
\text { from Land_Surface_Elev. }\end{array}$ \\
\hline County & County where the well is. \\
\hline Aquifer & Aquifer where the well is. \\
\hline Land_Surface_Elev & Land surface elevation where the well is. \\
\hline Bottom_Screen & Bottom-screen interval depth. \\
\hline Longitude & Longitude where the well is. \\
\hline Latitude & Latitude where the well is. \\
\hline Recorder & Identifies if the well is a continuous recorder well. \\
\hline
\end{tabular}

${ }^{1}$ All elevations are in feet and use the North American Vertical Datum of 1988.

identify the user-specified location of the model run and the wells used in the analysis are produced.

The summary file runname_analysis.html, called the dashboard, is a static report that contains a summary of output from the NDakGWtool for the user-specified location. In the gisdata function, the analyses.Rmd script is called to write the dashboard (table 1, fig. 6, appendix). The analyses.Rmd script uses the flexdashboard R package (table 1) and, for a given set of user-specified arguments, a static dashboard containing nine tabs is generated. The first tab is a map showing different layers: model domain, glacial drift aquifers, all wells in the NDSWC database, and wells used in the analysis (fig. 6A). The second tab is a summary table of the estimated monthly mean groundwater elevations, standard error of estimated groundwater elevations, and estimated depth of groundwater below land-surface datum for the user-specified location (fig. 6B). The remaining tabs, one for each of the 7 months, contain three maps of the grid cell containing the user-specified location: estimated monthly mean groundwater surface and standard error, and depth of groundwater below land-surface datum (fig. $6 \mathrm{C}$ ). Note, only the month of April is shown on figure $6 C$. Similar to the maps produced in the 


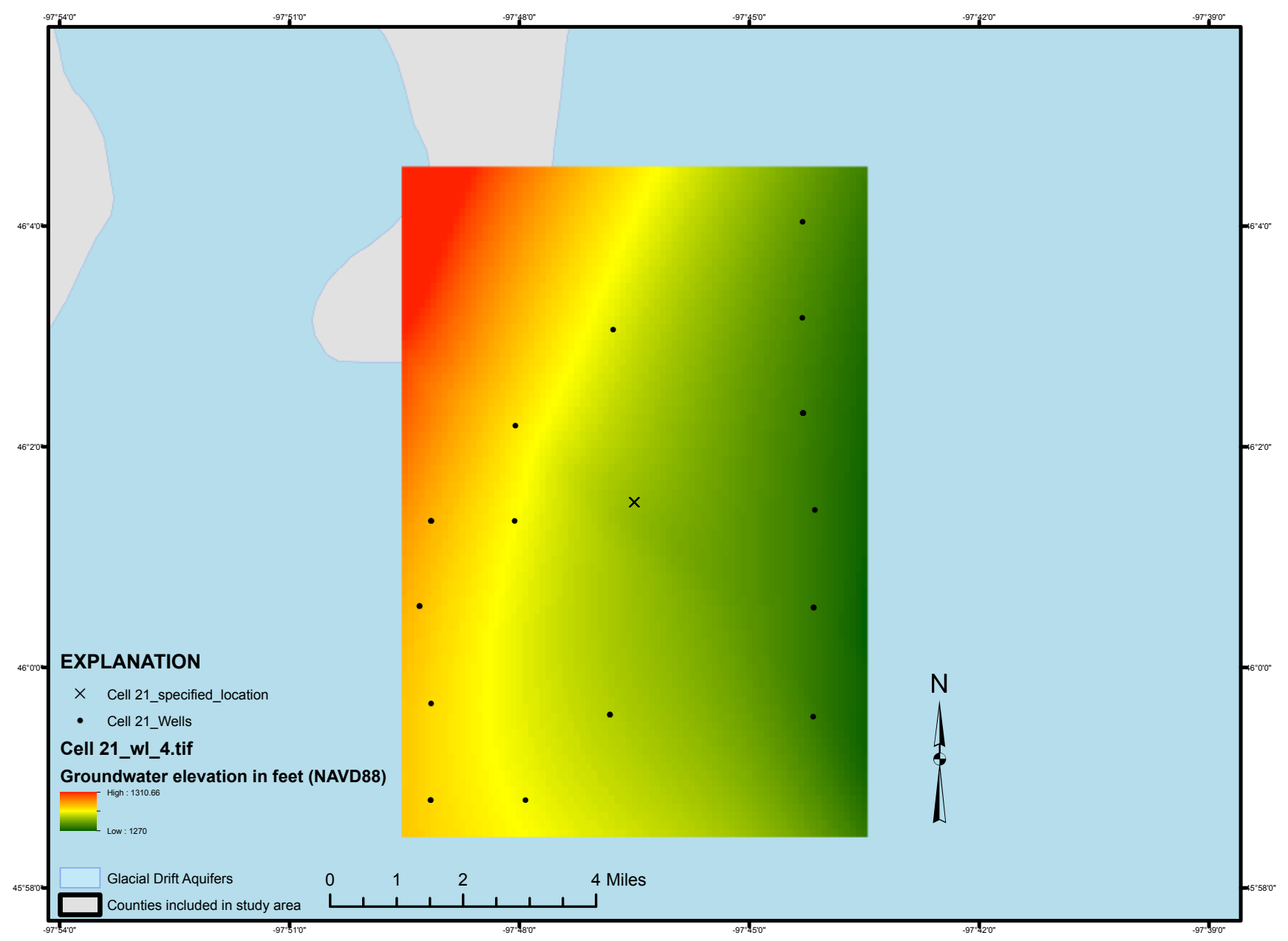

Figure 5. Example output from the NDakGWtool of ArcGIS files for Cell 21: Cell 21_specified_location, shape file layer that identifies the user-specified location; Cell 21_Wells, shape file layer that identifies wells used in the analysis, and Cell 21_wl_4.tif is the raster image of estimated groundwater surface for April.

diagnostic files, output is for the 0.1 - by 0.1 -degree cell and the $\mathrm{X}$ indicates the user-specified location for the model run (latsp, lonsp) and circles representing the locations of wells included in the analysis.

\section{Model Testing}

Model testing was performed by evaluating diagnostic output of the model for the center point of each grid cell, and through model testing, the model domain was established. During model testing, the wlmodelCell function could be executed for any location in the study area, but once the model domain was established, results were only available for the model domain. The wlmodelCell function was executed for the center point of each of the 5,340 grid cells for the 32-county study area using groundwater elevations from 1995 to 2015. The more recent (1995-2015) period represents a period of wetter conditions and generally higher groundwater elevations in central and eastern North Dakota compared to earlier years. The coverage of the tool was narrowed down through 2 main steps to include 413 grid cells in the model domain. First, most grid cells were eliminated because they did not meet the minimum requirements of sufficient data as determined in the wlmodelcell function (data requirements are described in the "Statistical Model for Estimating Groundwater Elevations" section). Many of these grid cells had no data because they were not overlying areas of glacial drift aquifers. Grid cells that were not in designated areas of glacial drift aquifers were initially included to address the need of the NRCS to include as many shallow groundwater areas as possible. Other grid cells in designated areas of glacial drift aquifers that may have had sufficient observation wells had measurement frequencies that were too sparse, a period of record that was too short, or well coverage that was too sparse and inconsistent to produce 


\section{A}

USGS Interactive Tool to Estimate Groundwater Elevations in Central and Eastern North Dakota

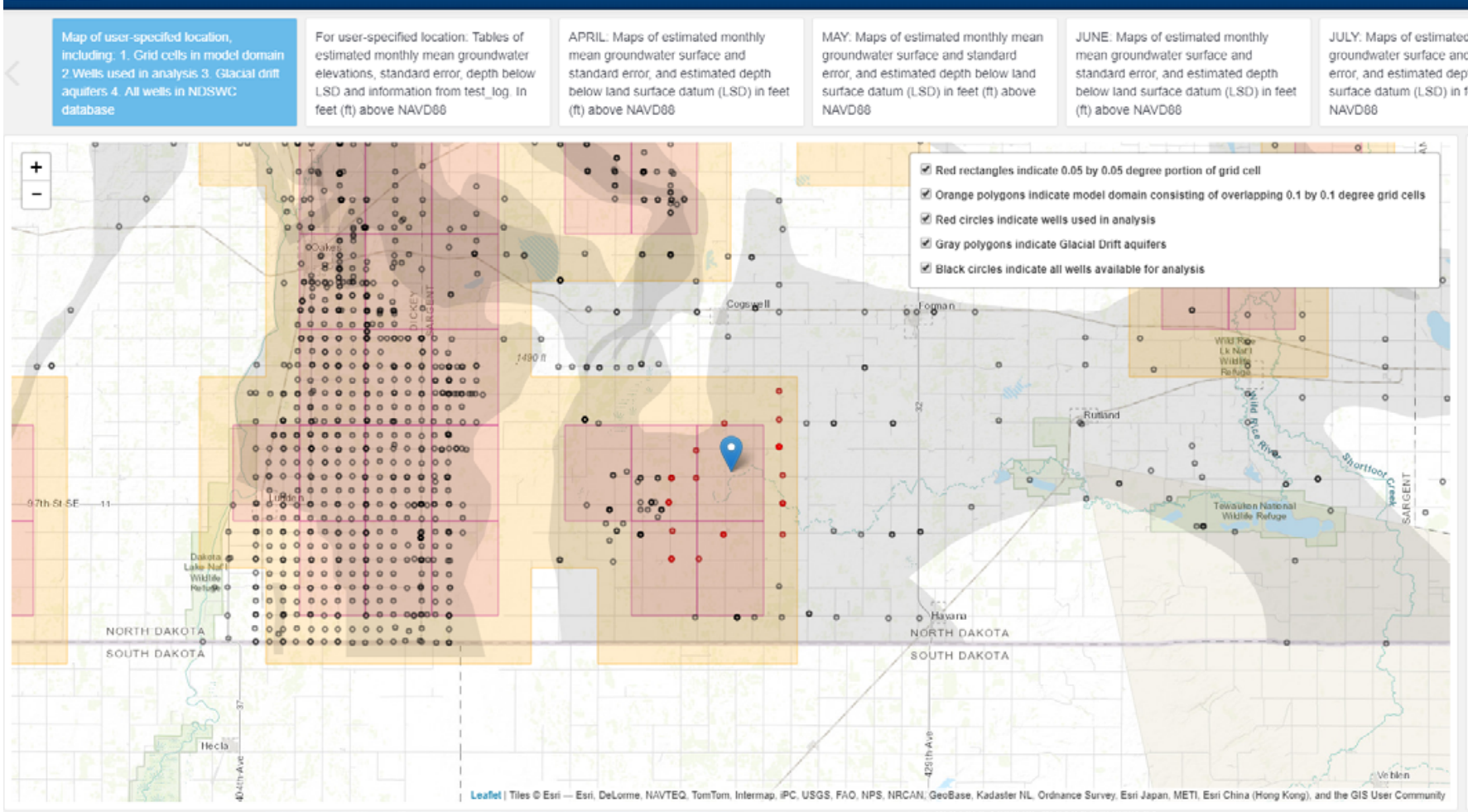

$\boldsymbol{B}$

USGS Interactive Tool to Estimate Groundwater Elevations in Central and Eastern North Dakota

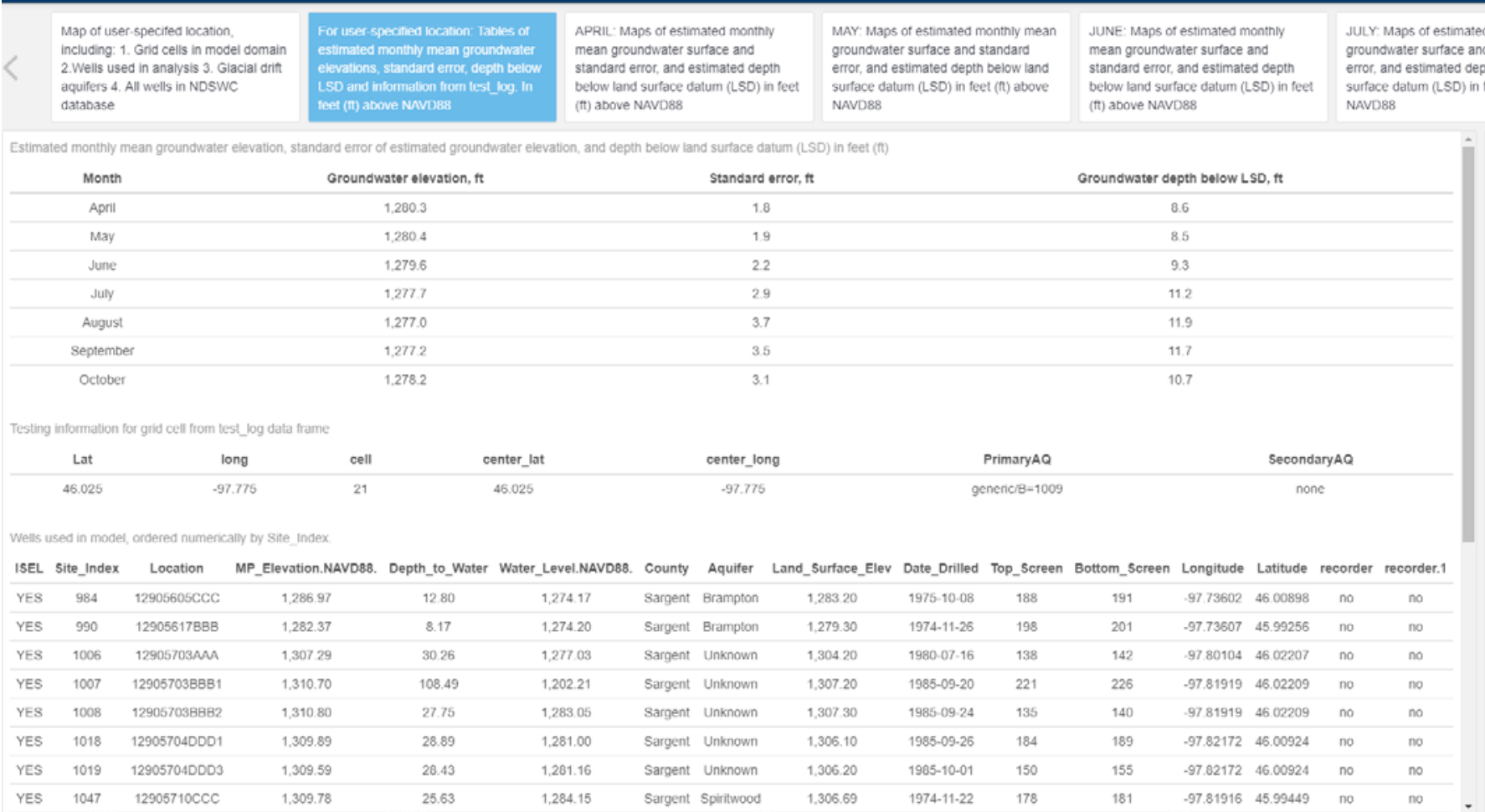

Figure 6. Screenshots from the NDakGWtool of the static summary file runname_analysis.html, called the dashboard, for Cell 21. $A$, The first tab of the dashboard identifies user-specified location and wells used in analysis. $B$, The second tab provides a summary table for each month. 


\section{C} USGS Interactive Tool to Estimate Groundwater Elevations in Central and Eastern North Dakota
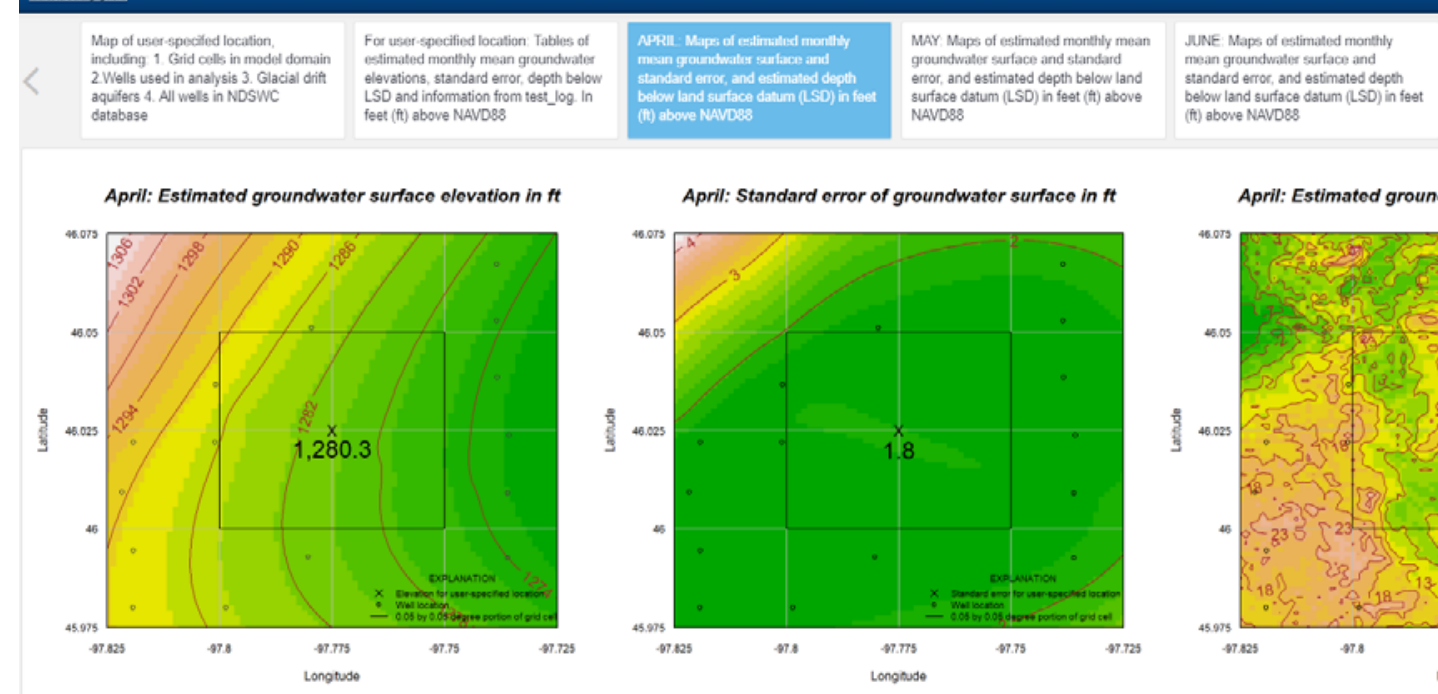
\begin{tabular}{l|l} 
JuLY Maps of estimated monthly mean & AUGUST \\
groundwater surface and standard \\
mean gros
\end{tabular} error, and estimated depth below land surface diand
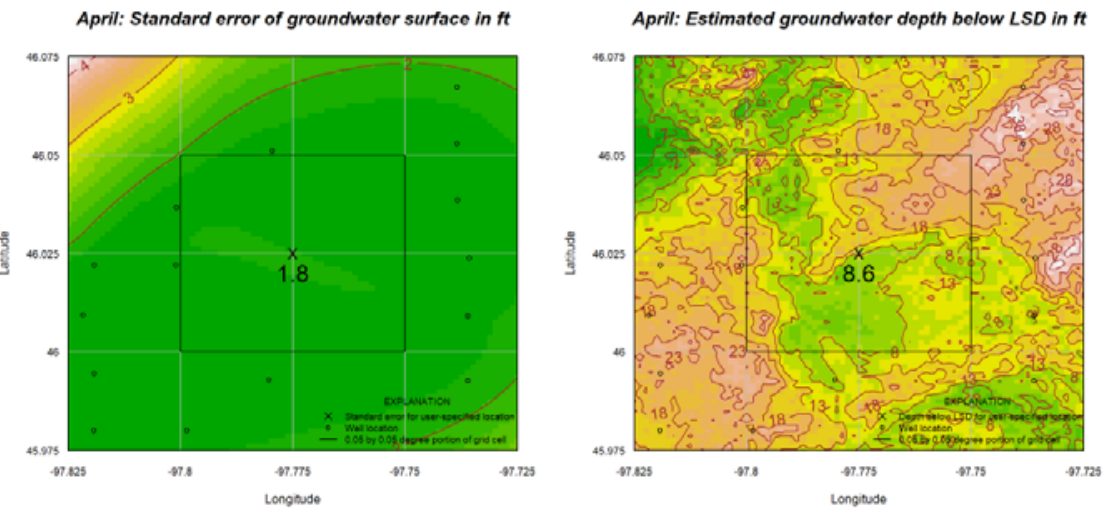

Figure 6. Screenshots from the NDakGWtool of the static summary file runname_analysis.html, called the dashboard, for Cell 21.-Continued. $C$, The third tab shows summary plots of estimated monthly mean groundwater surface and standard error and depth of groundwater below land surface, in feet, for April. Subsequent tabs are the same as the third tab but specific to each month.

reliable models. As a result, only 660 grid cells of the initial 5,340 cells contained sufficient data to produce reliable model results. Of those 660 cells, 413 were identified as having reliable output and were used to define the model domain (fig. 3). The model was deemed reliable through manual examination of diagnostic output to verify groundwater elevations generally were within the prediction limits, standard errors were relatively low (generally less than $10 \mathrm{ft}$ ), and the estimated maps seemed to represent a spatially homogeneous groundwater system. Results for the 413 cells in the model domain were manually examined, and modified as needed, to optimize accuracy and reliability based on diagnostic output to determine an appropriate default for the spaqname0 argument. In some cases, one or more alternate choices were appropriate for the spaqname 0 argument depending on the location or application being considered. The default and alternate values, if defined, for spaqname 0 are provided in the test_log data frame (table 1, appendix). In the test_log data frame, the default value is referred to as PrimaryAQ, and the alternate value is referred to as SecondaryAQ. If the spaqname0 argument is not specified by the user, the default value from test_log is used. The default results can be examined, and the spaqname 0 argument can be modified for the user's purpose. In some cases, the estimated groundwater surface may be highly uncertain, and there may be no apparent way to modify the spaqname0 argument to obtain more accurate results with the available data. Although the model domain was determined using the period 1995-2015, for locations with sufficient data availability, it could be used to estimate groundwater elevations for the earlier (1960-94) period, or for any user-specified period during 1960-2017. It is important to note that any variations in the arguments beyond the testing conditions may result in a model that is not reliable; thus, the user should closely assess the model output when specifying different values for arguments.

\section{Use of the Interactive Tool}

Depending upon the groundwater elevation data available for developing the model, uncertainty of the estimated groundwater elevations from the NDakGWtool can vary for the user-specified location. In this section, an example of results for a location with ample data and a location with sparse data available for developing a model are illustrated. Also, two examples are illustrated on how the user can use the spaqname0 argument to refine results depending on the user's purpose.

An example of a location with ample data available for developing the model is Cell 21 (fig. 4). From the test_log data frame, the default argument is defined as spaqname0 $=$ "generic $/ B=1009$ " (appendix). The modifier "generic" indicates wells with any aquifer name are being used and well 1009 is being removed using the B (spbadwells; table 2) modifier within the spaqname0 argument. Well 1009 was removed from the analysis for this cell because all groundwater elevations were outside the prediction limits. 
Investigation of the diagnostic files provides the user with a wealth of information about the data available to fit the model, the resulting model itself, and results from the model; for example, from runname wells.csv (fig. $4 A$, table 4), wells used in the model for Cell 21 , column ISEL $=$ YES, are in a mix of aquifers identified as Unknown, Spiritwood, or Brampton aquifers. Aquifers designated as "Unknown," "Undefined," and "Unnamed" are present throughout the dataset and indicate the well is in an aquifer that has not been identified for various reasons (Michael Ginsbach, NDSWC, oral commun., 2017). Based on the "Latitude" and "Longitude" columns, wells $1195,1196,32366$, and 32367 can be identified as being colocated. The "Date Drilled" column identifies wells 1195 and 1196 were drilled in 1981, but the aquifer name was not identified, and wells 32366 and 32367 were drilled in 2006 and identified to be in the Spiritwood aquifer. From inspection of the runname near.pdf file (not shown), groundwater elevations from wells 1195 and 1196 are no longer available after 2006, but wells 32366 and 32367 have groundwater elevations from 2006 to 2015. From these two diagnostic files, because the wells are of the same depth, it can be determined that wells 32366 and 32367 are a continuation of wells 1195 and 1196 , respectively. Because wells fail over time, wells are discontinued and new wells are drilled in about the same location and depth (Michael Ginsbach, NDSWC, oral commun., 2017). Valuable information for developing the model is included by using wells in the "Unknown" aquifer in the analysis.

Details about the model developed for Cell 21 are provided in the runname_fit.pdf file (fig. $4 C$ ). As mentioned in the "Statistical Model for Estimating Groundwater Elevations" section, the LWR model can be different for each month, depending on the availability of groundwater elevations. For the April model of Cell 21, 70 groundwater elevations were identified as outliers, but 531 groundwater elevations for 23 wells remained to develop the model (fig. 4C). The LWR model has a degree $=2$, span $=1$, indicating the density and distribution of wells are fairly evenly distributed and a relatively smooth groundwater surface is estimated. The runname_img. pdf file provides a more detailed view of the results and shows that, although the user-specified location is in an area where the closest wells are roughly $5 \mathrm{mi}$ ( 0.05 degrees) away, the estimated groundwater elevation for the user-specified location has a small standard error, less than $2 \mathrm{ft}$ (fig. 4D). Also, the colocated wells identified in the runname_wells.csv file are easily detected in the runname img.pdf file. This is an example of a grid cell for which using the default argument produces reliable results and would probably not require further modification.

There also are many cells in the model domain that have few wells and few groundwater elevations for developing the LWR model; for example, Cell 33 has only 5 wells and 103 groundwater elevations available to develop a model (fig. 7A). The resulting model is the simplest LWR form of degree $=1$ and span $=1$. Output is available for this grid cell, but standard errors are larger than what was seen in Cell 21 (fig. $7 B$ ).

The default argument for spaqname 0 specified in the test_log data frame can be used, but the user may have detailed knowledge of aquifer behavior and may want to modify the optional arguments to better match the conceptual understanding of the groundwater surface. A couple of examples are illustrated below. The first example is a situation in which two aquifers overlay one another within the same grid cell. In Cell 1, the Guelph aquifer overlays the Ellendale aquifer (fig. 8). By using the default argument from the test_log, spaqname0 = "default," wells in the Ellendale aquifer

\section{$\boldsymbol{A}$}

Cell 33 (latsp=46.125, lonsp=-100.225) - Model fit and estimated monthly mean groundwater surface and standard errors for Apr $1995-2015$
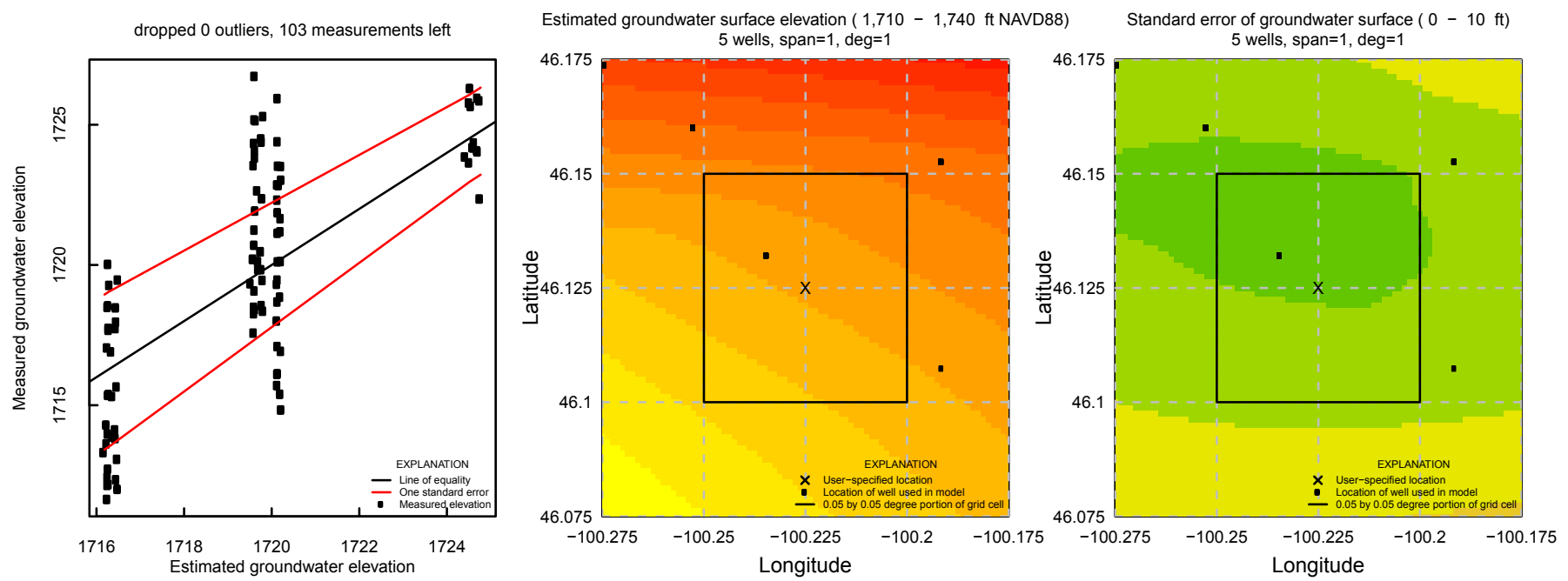

Figure 7. Screenshot from the NDakGWtool of diagnostic output for a user-specified location with sparse data. Arguments specified in wImodelcell function include lats $p=46.125$, lons $p=-100.225$, runname $=$ Cell 33, and spaqname0 = "default." $A$, runname_fit.pdf. 
$B$

Cell 33 (latsp=46.125, lonsp=-100.225) -Estimated monthly mean groundwater surface and standard errors for Apr $1995-2015$
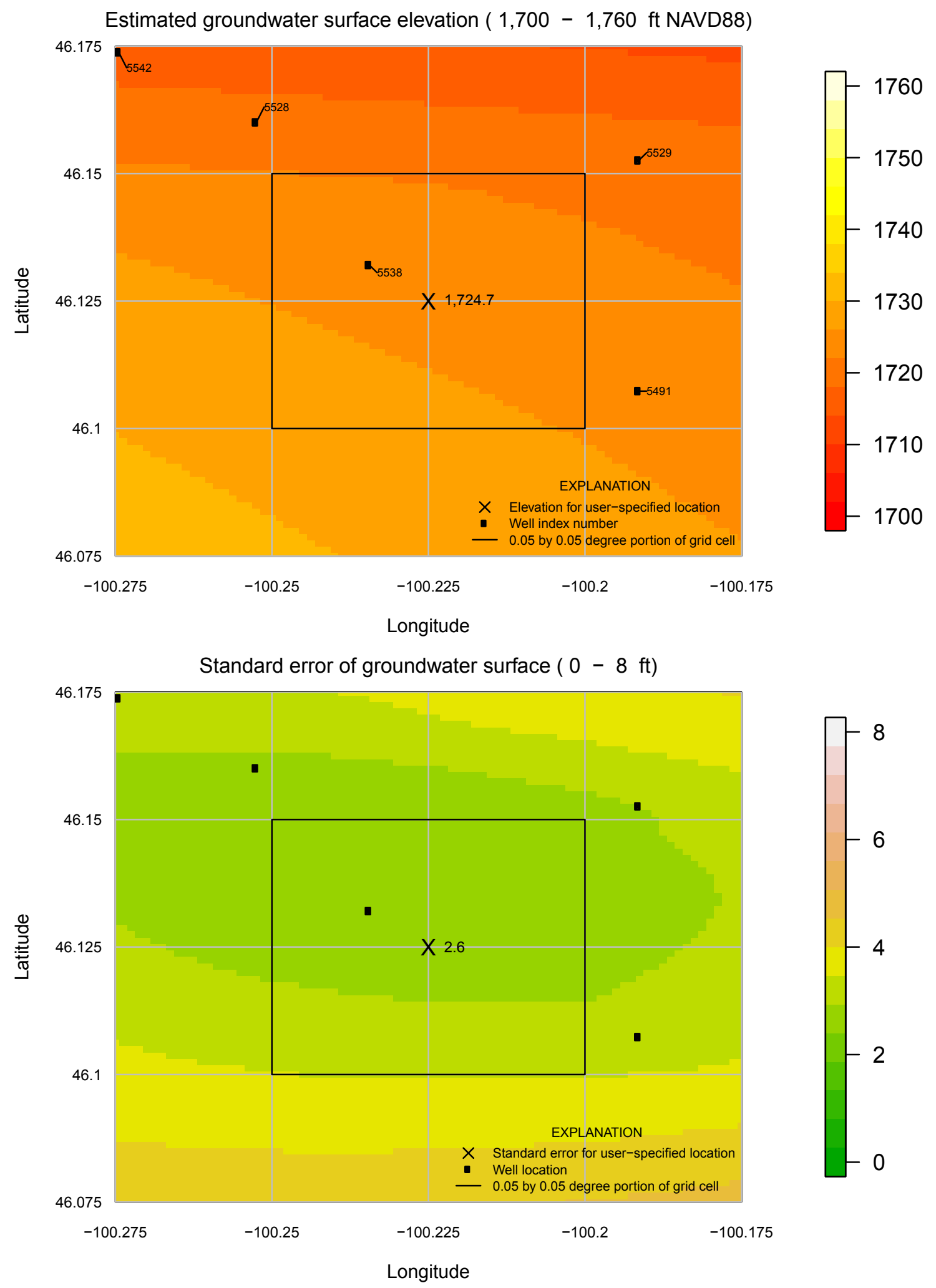

Figure 7. Screenshot from the NDakGWtool of diagnostic output for a user-specified location with sparse data.-Continued. $B$, runname_img.pdf. 
A USGS Interactive Tool to Estimate Groundwater Elevations in Central and Eastern North Dakota

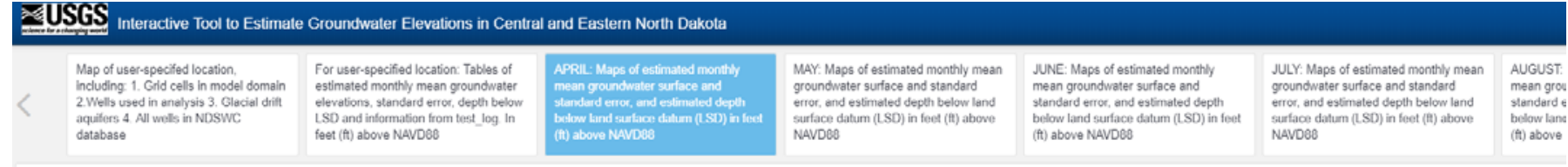
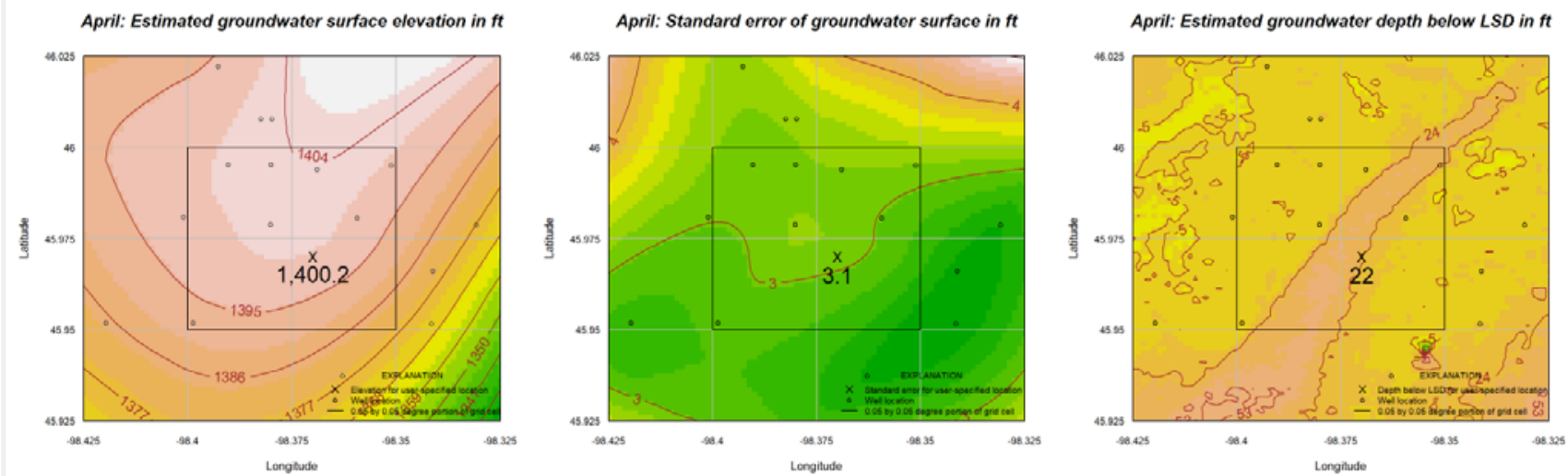

B
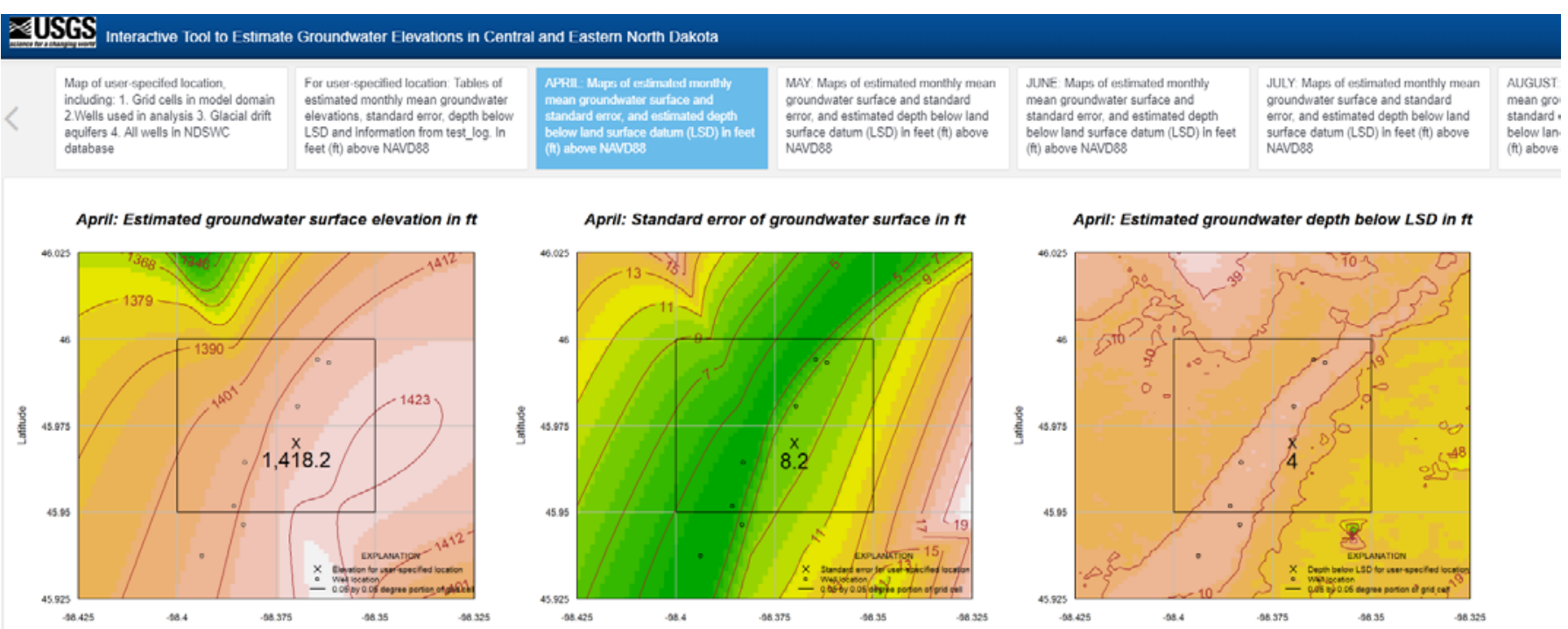

Figure 8. Screenshots from NDakGWtool to illustrate the modification of optional argument spaqname0. Arguments specified in the wImodelcell function include lats $p=45.97$, lons $p=-98.37$, runname $=$ Cell 1. $A$, spaqname $0=$ "default," default argument $=$ "Ellendale." $B$, spaqname0 = "Guelph."

are selected, and the April monthly mean groundwater elevation is $1,400.2 \mathrm{ft}$, standard error is $3.1 \mathrm{ft}$, and the depth below land surface is $22 \mathrm{ft}$ (fig. $8 A$ ). If the user is interested only in wells in the Guelph aquifer, the aqname modifier can be specified as "Guelph" in the spaqname0 argument (spaqname0 $=$ "Guelph"), and the results are different (fig. 8B). Using spaqname $0=$ "Guelph," the April monthly mean groundwater elevation is higher at $1,418.2 \mathrm{ft}$, and depth below land surface is $4 \mathrm{ft}$; however, the standard error of $8.2 \mathrm{ft}$ is larger because the Guelph aquifer has fewer wells with groundwater elevations (indicated by open circles) and a narrow, smaller spatial distribution of those wells in the Guelph aquifer.

The second example for Cell 20 demonstrates how the user can interactively refine output based on the well screen-interval depth (fig. 9). For Cell 20, in test log, the default argument for spaqname 0 for Cell 20 is specified as "generic/ $D>100$." Using the default argument, wells with a deeper screen-interval depth are used for the model, and the groundwater surface slopes from northwest to southeast across the cell. The monthly mean groundwater elevation for April 
$\boldsymbol{A}$ ZUSG Interactive Tool to Estimate Groundwater Elevations in Central and Eastern North Dakota
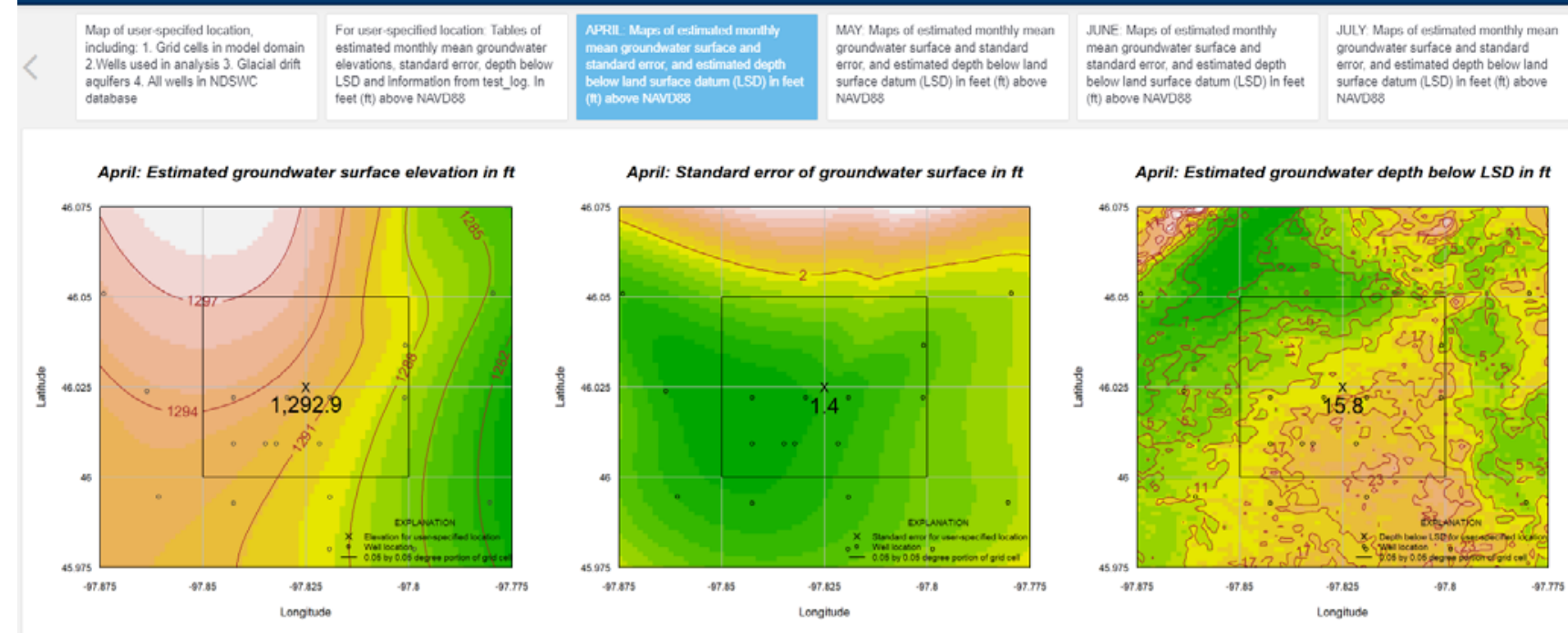

AUGusT Navoss (ז) above NAVDBS NAVDBS

April: Standard error of groundwater surface in $\mathrm{ft}$

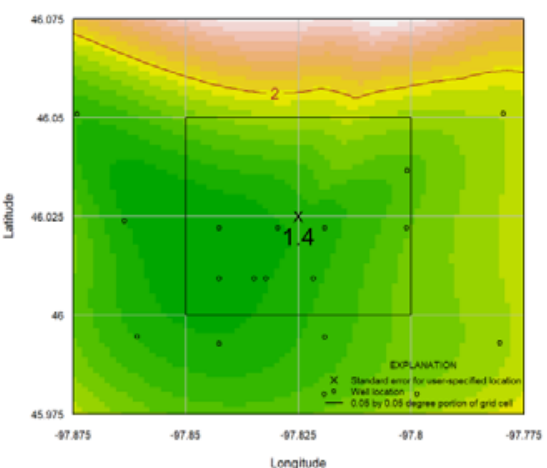

April: Estimated groundwater depth below LSD in ft

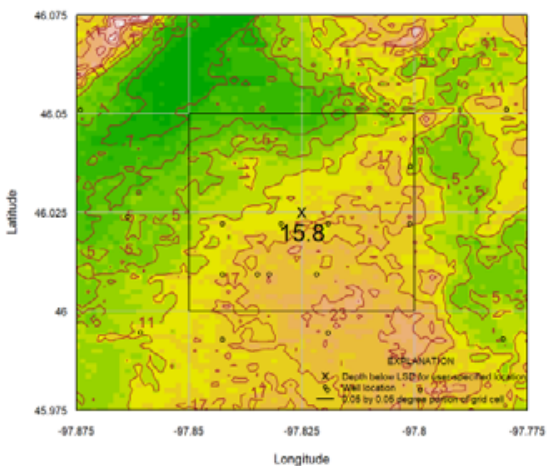

B \US Interactive Tool to Estimate Groundwater Elevations in Central and Eastern North Dakota

Map of user-speclifed location. induding 1 . Grid cells in model domahn aquifers 4. All wells in NDSWC aquifers 4 . Al wabase
For user-spectifed location: Tables of estimated monthly mean groundwater LSD and infomation from depth below feet (fi) above NAVDOO

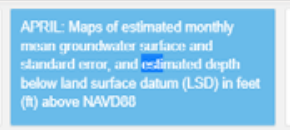

MAY: Maps of estimated monthly mean groundwater surface and standard surface and estimated depth below land
sutace in feet (ti) above NAVDOB
JUNE: Maps of estimated monthly standard error, and estirnated depth below land surface datum (LSO) in feet (t) above NAVDes
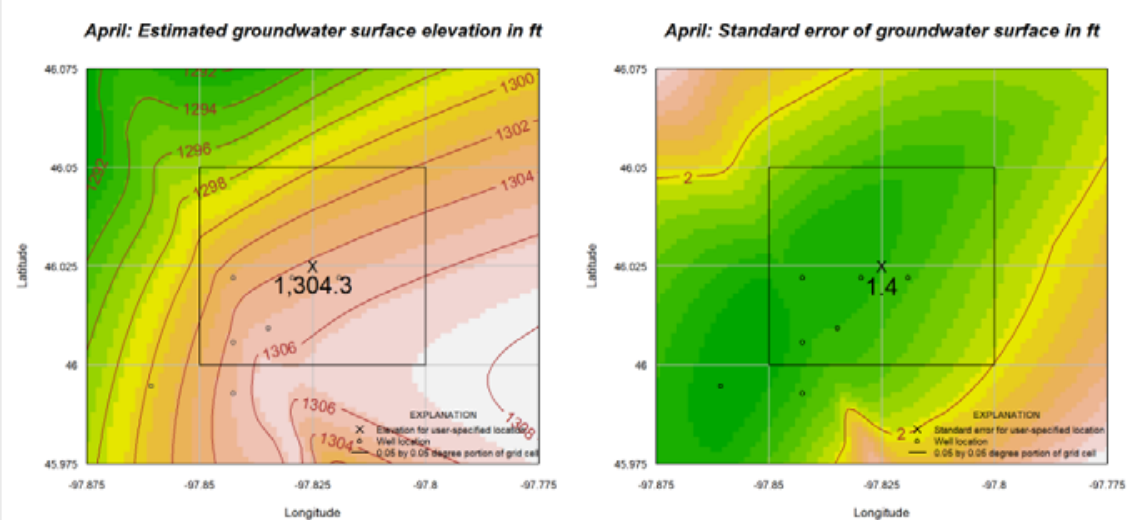

April: Estimated groundwater depth below LSD in $\mathrm{ft}$

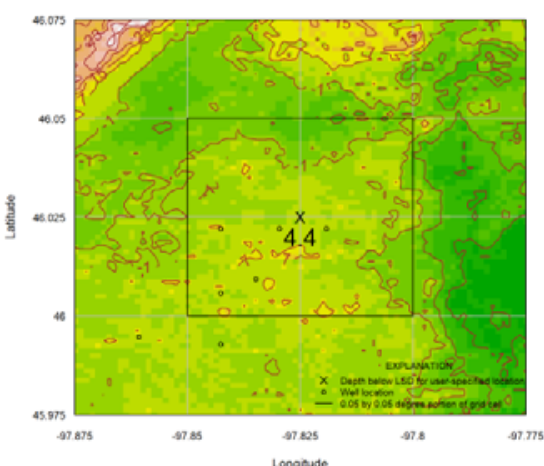

Figure 9. Example of output from the interactive groundwater tool to illustrate modification of an optional argument. Arguments specified in the wlmodelcell function include lats $p=46.025$, lons $p=-97.825$, and runname $=$ Cell 20 . $A$, spaqname0 = default; default argument $=$ "generic/D $>100$." $B$, spaqname $0=$ "generic $/ D<100 . "$

is $1,292.9 \mathrm{ft}$ (fig. $9 \mathrm{~A}$ ). If the user is only interested in wells open to the aquifer at a shallower depth, the modifier within the spaqname0 argument for screen-interval depth can be specified as $D<100$ (spdepth; table 2). Model results are much different, with the water surface sloping from southeast to northwest across the cell, and the monthly mean groundwater elevation for April is more than $11 \mathrm{ft}$ higher at $1,304.3 \mathrm{ft}$ (fig. 9B). For Cell 20, groundwater elevation results are different when wells are selected based on screen-interval depth. 


\section{References Cited}

Cleveland, W.S., Grosse, E., and Shyu, W.M., 1992, Local regression models, chap. 8 of Chambers, J.M., and Hastie, T.J., eds., Statistical models in S: Pacific Grove, Calif., Wadsworth and Brooks/Cole Advanced Books and Software, p. 309-376.

Esri, 2018, ArcGIS desktop: Esri software, accessed August 17, 2015, at https://desktop.arcgis.com/en/desktop/.

North Dakota State Water Commission, 2017, Ground/surface water data: North Dakota State Water Commission web page, accessed July 21, 2017, at http://www.swc.nd.gov/ info_edu/map_data_resources/groundsurfacewater/.

R Development Core Team, 2018, R-A language and environment for statistical computing: Vienna, Austria, R Foundation for Statistical Computing, accessed March 23, 2018, at https://www.r-project.org.

U.S. Department of Agriculture, 2015, Hydrology tools for wetland identification and analysis, chap. 19 of Engineering field handbook: Washington D.C., U.S. Department of Agriculture, Natural Resources Conservation Service 113 p.

U.S. Geological Survey, 2017, National Elevation Dataset: U.S. Geological Survey web page, accessed July 24, 2017, at https://nationalmap.gov/elevation.html. 


\section{Appendix. R Documentation}

A zipped folder, NDakGWtool.zip, contains the following:

- Rscripts_-model_run.R, analyses.Rmd;

- R objects_-obs_wl, test_log, lse;

- files_-wlinputalldata.R, wlmodelCell.R, model_data. Rdata, gisdata.R, sites.txt, water_levels.txt, USGS_ID_ black.png, readme.txt; and

- folders-GIS, OUTPUT.

The folder can be downloaded at https://doi.org/10.3133/ofr20181185.

For more information about this publication, contact:

Director, USGS Dakota Water Science Center

821 East Interstate Avenue

Bismarck, ND 58503

701-250-7400

For additional information, visit: https://www.usgs.gov/centers/ dakota-water

Publishing support provided by the

Rolla Publishing Service Center 



\section{$\frac{\mathbb{3}}{\mathrm{C}}$}

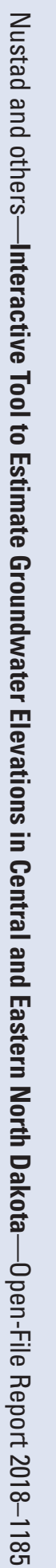

